\title{
Mixing rank-one actions of locally compact Abelian groups
}

\author{
Alexandre I. Danilenko ${ }^{\mathrm{a}, 1}$, Cesar E. Silva ${ }^{\mathrm{b}, *}$ \\ a Max Planck Institute of Mathematics, Vivatsgasse 7, D53111 Bonn, Germany \\ b Department of Mathematics, Williams College, Williamstown, MA 01267, USA
}

Received 26 April 2005; received in revised form 3 April 2006; accepted 30 May 2006

Available online 15 December 2006

\begin{abstract}
Using techniques related to the $(C, F)$-actions we construct explicitly mixing rank-one (by cubes) actions $T$ of $G=\mathbb{R}^{d_{1}} \times \mathbb{Z}^{d_{2}}$ for any pair of non-negative integers $d_{1}, d_{2}$. It is also shown that $h\left(T_{g}\right)=0$ for each $g \in G$.

๑) 2006 Elsevier Masson SAS. All rights reserved.
\end{abstract}

\section{Résumé}

En utilisant des techniques liées aux action $(C, F)$, nous construisons explicitement des actions mélangeantes de rang un (par cubes), $T$ de $G=\mathbb{R}^{d_{1}} \times \mathbb{Z}^{d_{2}}$ pour toute paire de nombres entiers $d_{1}, d_{2} \geqq 0$. On prouve aussi que $h\left(T_{g}\right)=0$ pour chaque $g \in G$. (c) 2006 Elsevier Masson SAS. All rights reserved.

MSC: 37A25; 37A 15

Keywords: Ergodic action; Mixing; Rank-one action; Entropy

\section{Introduction}

Mixing rank-one transformations (and actions of more general groups) have been of interest in ergodic theory since 1970 when Ornstein constructed an example of mixing transformation without square root [18]. His method was used later as the core of a number of other remarkable constructions (see $[20,21,13,10,17,19,8]$, etc.) Since then the dynamical properties of mixing rank-one transformations have been deeply investigated. It is now well known that such transformations are mixing of all orders [14,22] and have minimal self-joinings of all orders [15,22]. This implies in turn that they are prime and have trivial centralizer [21]. The results on multiple mixing were extended to rank-one mixing actions of $\mathbb{R}^{d}$ and $\mathbb{Z}^{d}$ [22-24] and to rank-one mixing actions of a wide class of discrete countable Abelian groups having an element of infinite order [11].

\footnotetext{
This project was supported in part by the NSF under the COBASE program, contract INT-0002341. Also, the first named author was supported in part by CRDF, grant UM1-2546-KH-03.

* Corresponding author.

E-mail addresses: danilenko@ilt.kharkov.ua (A.I. Danilenko), csilva@williams.edu (C.E. Silva).

1 Permanent address: Institute for Low Temperature Physics \& Engineering of Ukrainian National Academy of Sciences, 47 Lenin Ave., Kharkov, 61164, Ukraine.
} 
Despite this progress, there are not many concrete examples of rank-one mixing actions that are known. Most of them were obtained via stochastic cutting-and-stacking techniques using "random spacers". Ornstein initiated this technique in [18], and more recent generalizations include the constructions of $\mathbb{R}$-actions in [19] and actions of infinite sums of finite groups in [8], as well as the del Junco-Madore actions of Abelian extensions of $\mathbb{Z}^{d}$ by locally finite groups $[10,17]$. The latter actions were only shown to be weakly mixing but conjectured to be mixing in [17]. While demonstrating the existence of mixing rank-one actions (which is a non-trivial problem!), these works do not exhibit a specific such transformation or action. In 1992 Adams and Friedman [2] gave a non-random algorithm that leads to a mixing rank-one construction. Using the ideas from that manuscript Adams [1] proved in 1998 the old conjecture that the classical staircase is mixing. That gave the first explicit example of mixing cutting-and-stacking transformation. Higher dimensional mixing staircase $\mathbb{Z}^{d}$-actions were later constructed in [3]. We note that the complete proof of the fact that they are mixing was given there only in dimension $d=2$. As one of the consequences of our work, we complete the proof for all $d>2$ (see Remark 4.12 below). Recently, a more general family of mixing "polynomial" staircase $\mathbb{Z}$-actions was constructed in [5]. Another interesting non-random construction appears in a recent work [12] devoted to smooth realizations of mixing rank-one flows on the 3-torus.

Our main purpose here is to construct explicitly a family of mixing rank-one actions of $\mathbb{R}^{d_{1}} \times \mathbb{Z}^{d_{2}}$ for all nonnegative $d_{1}$ and $d_{2}$. It seems plausible that Orntein's stochastic method also can be adapted to produce mixing rankone actions of these groups. We note however that our construction is more general and the 'randomness' can be incorporated into it (see [5] and [8] for a detailed discussion on that for $\mathbb{Z}$-actions and actions of infinite sums of finite groups respectively). Moreover, the main advantage of our approach is that the examples in our family are 'absolutely concrete', i.e., the parameters in the construction are all explicitly specified—the 'spacer mappings' are polynomials with known coefficients.

As a corollary we show that this family includes all the examples of mixing rank-one $\mathbb{Z}^{d}$-actions constructed previously in [1,3] and [5]. Our approach is based on ideas that first appeared in those three works. However, in this paper we proceed entirely in the framework of $(C, F)$-actions for locally compact second countable (l.c.s.c.) Abelian groups, and in fact we develop a large part of the theory in the more general context of these actions. In particular, we encounter here some new problems that are specific to higher dimensions and the continuity of the groups. Recall that the $(C, F)$-construction of finite measure-preserving actions of discrete countable amenable groups appeared in [10] as an algebraic counterpart of the "geometrical" cutting-and-stacking method developed for $\mathbb{Z}$-actions. Later it was used (in a modified form) by the authors in the framework of infinite measure-preserving and non-singular countable Abelian group actions, as a convenient tool for modeling examples and counterexamples with various properties of weak mixing and multiple recurrence (see $[6,7,9])$.

Let $G$ be a non-compact 1.c.s.c. Abelian group and $T=\left(T_{g}\right)_{g \in G}$ a measurable action of $G$ on a standard probability space $(X, \mathfrak{B}, \mu)$.

Definition 0.1. $T$ is said to be mixing if for all subsets $A, B \in \mathfrak{B}$ we have

$$
\lim _{g \rightarrow \infty} \mu\left(T_{g} A \cap B\right)=\mu(A) \mu(B) .
$$

A sequence $g_{n} \rightarrow \infty$ in $G$ is called mixing if (0.1) holds along $g_{n}$ as $n \rightarrow \infty$.

Notice that an action is mixing whenever each sequence converging to infinity in $G$ contains a mixing subsequence.

\section{Definition 0.2.}

(i) A Rokhlin tower or column for $T$ is a triple $(Y, f, F)$, where $Y \in \mathfrak{B}, F$ is a relatively compact subset of $G$ and $f: Y \rightarrow F$ is a measurable mapping such that for any Borel subset $H \subset F$ and an element $g \in G$ with $g+H \subset F$, one has $f^{-1}(g+H)=T_{g} f^{-1}(H)$.

(ii) We say that $T$ is of funny rank-one if there exists a sequence of Rokhlin towers $\left(Y_{n}, f_{n}, F_{n}\right)$ such that $\lim _{n \rightarrow \infty} \mu\left(Y_{n}\right)=1$ and for any subset $B \in \mathfrak{B}$, there is a sequence of Borel subsets $H_{n} \subset F_{n}$ such that

$$
\lim _{n \rightarrow \infty} \mu\left(B \Delta f_{n}^{-1}\left(H_{n}\right)\right)=0 .
$$

(iii) If $G=\mathbb{R}^{d_{1}} \times \mathbb{Z}^{d_{2}}, T$ is of funny rank-one and, in addition, the subsets $F_{n}$ from (ii) are as follows

$$
F_{n}=\left\{\left(t_{1}, \ldots, t_{d_{1}+t_{2}}\right) \in G \mid 0 \leqslant t_{i}<a_{n} \text { for all } i=1, \ldots, d_{1}+d_{2}\right\}
$$


for some $a_{n} \in \mathbb{R}, n=1,2, \ldots$, then we say that $T$ is of rank-one (or rank-one by cubes).

It is easy to see that any funny rank-one action is ergodic.

Note that what we call funny rank-one is called rank-one by del Junco and Yassawi in case $G$ is discrete and countable and $G \neq \mathbb{Z}[11]$; also del Junco and Yassawi require in addition that the sequence of sets $\left(F_{n}\right)_{n=1}^{\infty}$ is a Følner sequence.

The paper is organized as follows. In Section 1 we extend the concept of $(C, F)$-action introduced for countable discrete groups (see [10,6]) to the class of 1.c.s.c. Abelian ones. A special family of actions, whose mixing properties will be under investigation in subsequent sections, is defined. In Section 2 we introduce a concept of uniformly mixing sequence and prove a fundamental lemma (Lemma 2.2) linking the uniform mixing along some special sequences with Cesàro means for the 'spacer mappings'. Then we find a sufficient condition for the total ergodicity of the actions under considerations. We also start to check the uniform mixing property for some special sequences. In particular, we show that if a sequence is of 'moderate growth' relative to a fixed Følner sequence in $G$ then (under some extra conditions on $G$ and the action) it is uniformly mixing (Lemma 2.9). Section 3 is devoted to the actions with restricted growththe property which was phrased explicitly in [5] for $G=\mathbb{Z}$ but used already in [1] and [3] in an implicit form. We note that our definition of restricted growth differs from that introduced in [5] (the latter does not extend from $\mathbb{Z}$ to arbitrary 1.c.s.c. Abelian group actions). However, they are equivalent for polynomial staircase actions. Theorem 3.5 provides a sufficient condition for the $(C, F)$-actions with restricted growth to be mixing. We also include here a couple of statements (Lemmas 3.9-3.11) facilitating verification of this condition for the $\mathbb{R}^{d_{1}} \times \mathbb{Z}^{d_{2}}$-actions to be constructed in the next section. Section 4 contains the main results of the paper: Theorems $4.9-4.11$ and 4.13 which provide families of mixing rank-one actions of $\mathbb{R}^{d}$ with $d>1, \mathbb{R}, \mathbb{Z}^{d}$ and $\mathbb{R}^{d_{1}} \times \mathbb{Z}^{d_{2}}$ respectively. Every such action is determined completely by a sequence of positive integers $\left(r_{n}\right)_{n=1}^{\infty}$ (corresponding to the sequence of 'cuts' in the cutting-andstacking construction) and a sequence $\left(s_{n}\right)_{n=1}^{\infty}$ of 'monotonic' polynomials of $d_{1}+d_{2}$ variables (corresponding to the sequence of 'spacer's maps' on the $n$-th step). The sequences are chosen in the following way: $\left(r_{n}\right)_{n=1}^{\infty}$ is any sequence of sub-exponential growth with $\lim _{n \rightarrow \infty} r_{n}=\infty$ and $\left(s_{n}\right)_{n=1}^{\infty}$ consists of some specially selected quadratic polynomials from Example 4.2. Moreover, if $d_{1} \neq 1$ (and only in this case) then $\left(s_{n}\right)_{n=1}^{\infty}$ can be chosen constant. If $d_{1}=1$ then $\left(s_{n}\right)_{n=1}^{\infty}$ can be chosen consisting of two alternating polynomials. Furthermore, using our techniques plus the Hilbertian van der Corput trick we can also treat a more complicated case where $\left(s_{n}\right)_{n=1}^{\infty}$ consists of polynomials of degree $>2$ (see Proposition 4.14). Example 4.15 provides a family of rank-one mixing transformations including the polynomial staircases from [5]. In the final section (Section 5) we show that the actions constructed in Section 4 have 'very weak' stochastic properties - the entropy of any individual transformation from such actions is zero. This fact holds for any rank-one (by cubes) action. However, it is no longer true for a more general class of actions of rank-one 'by rectangles' (see [20] for a counterexample).

\section{1. $(C, F)$-actions of locally compact Abelian groups}

In this section we introduce the $(C, F)$-actions of 1.c.s.c. Abelian groups and specify a subclass of them (see Definitions 1.2 and 1.4). We explain how the classical cutting-and-stacking transformations are included into this subclass (Remark 1.6). The aim of the paper is to show that this subclass contains mixing actions.

Let $G$ be a 1.c.s.c. Abelian group. Denote by $\lambda_{G}$ a $(\sigma$-finite) Haar measure on it. Given two subsets $E, F \subset G$, by $E+F$ we mean their algebraic sum, i.e., $E+F=\{e+f \mid e \in E, f \in F\}$. The algebraic difference $E-F$ is defined in a similar way. We hope that the reader will not confuse it with the set theoretical difference $E \backslash F$. If $E$ is a singleton, say $E=\{e\}$, then we will write $e+F$ for $E+F$. If $(E-E) \cap(F-F)=\{0\}$ then $E$ and $F$ are called independent. For an element $g \in G$ and a subset $E \subset G$, we set $E(g)=E \cap(E-g)$.

To define a $(C, F)$-action of $G$ we need two sequences $\left(F_{n}\right)_{n \geqslant 0}$ and $\left(C_{n}\right)_{n>0}$ of subsets in $G$ such that the following hold

$F_{0} \subset F_{1} \subset F_{2} \subset \cdots$ is a Følner sequence in $G$,

$C_{n}$ is finite and $\# C_{n}>1$,

$F_{n}+C_{n+1} \subset F_{n+1}$,

$F_{n}$ and $C_{n+1}$ are independent. 
We put $X_{n}:=F_{n} \times \prod_{k>n} C_{k}$, endow $X_{n}$ with the standard product Borel $\sigma$-algebra and define a Borel embedding $X_{n} \rightarrow X_{n+1}$ by setting

$$
\left(f_{n}, c_{n+1}, c_{n+2}, \ldots\right) \mapsto\left(f_{n}+c_{n+1}, c_{n+2}, \ldots\right) .
$$

Then we have $X_{1} \subset X_{2} \subset \cdots$. Hence $X:=\bigcup_{n} X_{n}$ endowed with the natural Borel $\sigma$-algebra, say $\mathfrak{B}$, is a standard Borel space. Given a Borel subset $A \subset F_{n}$, we denote the set

$$
\left\{x \in X \mid x=\left(f_{n}, c_{n+1}, c_{n+2} \ldots\right) \in X_{n} \text { and } f_{n} \in A\right\}
$$

by $[A]_{n}$ and call it an $n$-cylinder. It is clear that the $\sigma$-algebra $\mathfrak{B}$ is generated by the family of all cylinders.

Now we are going to define a measure on $(X, \mathfrak{B})$. Let $\kappa_{n}$ stand for the equidistribution on $C_{n}$ and $v_{n}:=$ $\left(\# C_{1} \ldots \# C_{n}\right)^{-1} \lambda_{G}\left\lceil F_{n}\right.$ on $F_{n}$. We define a product measure $\mu_{n}$ on $X_{n}$ by setting

$$
\mu_{n}=v_{n} \times \kappa_{n+1} \times \kappa_{n+2} \times \cdots,
$$

$n \in \mathfrak{N}$. Then the embeddings (1.5) are all measure preserving. Hence a $\sigma$-finite measure $\mu$ on $X$ is well defined by the restrictions $\mu\left\lceil X_{n}=\mu_{n}, n \in \mathbb{N}\right.$. To put it in another way, $(X, \mu)=\operatorname{inj} \lim _{n}\left(X_{n}, \mu_{n}\right)$. Since

$$
\mu_{n+1}\left(X_{n+1}\right)=\frac{v_{n+1}\left(F_{n+1}\right)}{v_{n+1}\left(F_{n}+C_{n+1}\right)} \mu_{n}\left(X_{n}\right)=\frac{\lambda_{G}\left(F_{n+1}\right)}{\lambda_{G}\left(F_{n}\right) \# C_{n+1}} \mu_{n}\left(X_{n}\right),
$$

it follows that $\mu$ is finite if and only if

$$
\prod_{n=0}^{\infty} \frac{\lambda_{G}\left(F_{n+1}\right)}{\lambda_{G}\left(F_{n}\right) \# C_{n+1}}<\infty, \quad \text { i.e., } \quad \sum_{n=0}^{\infty} \frac{\lambda_{G}\left(F_{n+1} \backslash\left(F_{n}+C_{n+1}\right)\right)}{\lambda_{G}\left(F_{n}\right) \# C_{n+1}}<\infty .
$$

For the rest of the paper we will assume that (1.6) is satisfied. Moreover, we choose (i.e., normalize) $\lambda_{G}$ in such a way that $\mu(X)=1$.

To construct a measure-preserving action of $G$ on $(X, \mu)$, we fix a filtration $K_{1} \subset K_{2} \subset \cdots$ of $G$ by compact subsets. Thus $\bigcup_{m=1}^{\infty} K_{m}=G$. Given $n, m \in \mathbb{N}$, we set

$$
D_{m}^{(n)}:=\left(\bigcap_{k \in K_{m}}\left(F_{n}-k\right) \cap F_{n}\right) \times \prod_{k>n} C_{k} \subset X_{n}
$$

and

$$
R_{m}^{(n)}:=\left(\bigcap_{k \in K_{m}}\left(F_{n}+k\right) \cap F_{n}\right) \times \prod_{k>n} C_{k} \subset X_{n} .
$$

It is easy to verify that

$$
D_{m+1}^{(n)} \subset D_{m}^{(n)} \subset D_{m}^{(n+1)} \quad \text { and } \quad R_{m+1}^{(n)} \subset R_{m}^{(n)} \subset R_{m}^{(n+1)} .
$$

We define a Borel mapping

$$
K_{m} \times D_{m}^{(n)} \ni(g, x) \mapsto T_{m, g}^{(n)} x \in R_{m}^{(n)}
$$

by setting for $x=\left(f_{n}, c_{n+1}, c_{n+2}, \ldots\right)$,

$$
T_{m, g}^{(n)}\left(f_{n}, c_{n+1}, c_{n+2}, \ldots\right):=\left(g+f_{n}, c_{n+1}, c_{n+2}, \ldots\right) .
$$

Now let $D_{m}:=\bigcup_{n=1}^{\infty} D_{m}^{(n)}$ and $R_{m}:=\bigcup_{n=1}^{\infty} R_{m}^{(n)}$. Then a Borel mapping

$$
K_{m} \times D_{m} \ni(g, x) \mapsto T_{m, g} x \in R_{m}
$$

is well defined by the restrictions $T_{m, g} \uparrow D_{m}^{(n)}=T_{m, g}^{(n)}$ for $g \in K_{m}$ and $n \geqslant 1$. It is easy to see that $D_{m} \supset D_{m+1}$, $R_{m} \supset R_{m+1}$ and $T_{m, g}\left\lceil D_{m+1}=T_{m+1, g}\right.$ for all $m$. It follows from (1.1) that $\mu_{n}\left(D_{m}^{(n)}\right) \rightarrow 1$ and $\mu_{n}\left(R_{m}^{(n)}\right) \rightarrow 1$ as $n \rightarrow \infty$. Hence $\mu\left(D_{m}\right)=\mu\left(R_{m}\right)=1$ for all $m \in \mathbb{N}$. Finally we set $\widehat{X}:=\bigcap_{m=1}^{\infty} D_{m} \cap \bigcap_{m=1}^{\infty} R_{m}$ and define a Borel mapping

$$
T: G \times \widehat{X} \ni(g, x) \rightarrow T_{g} x \in \widehat{X}
$$

by setting $T_{g} x:=T_{m, g} x$ for some (and hence any) $m$ such that $g \in K_{m}$. It is clear that $\mu(\widehat{X})=1$. 
Proposition 1.1. $T=\left(T_{g}\right)_{g \in G}$ is a free Borel measure preserving action of $G$ on a conull subset of the standard probability space $(X, \mathfrak{B}, \mu)$. It is of funny rank-one.

Proof. It suffices to verify only the latter claim. According to Definition 0.2 we have to find a sequence of Rokhlin towers 'appoximating' the dynamical system. Let $s_{n}$ denote the projection of $X_{n}=F_{n} \times C_{n+1} \times \cdots$ onto the first coordinate. It is easy to see that the sequence $\left(X_{n}, s_{n}, F_{n}\right)$ is as desired.

Throughout the paper we will not distinguish between two measurable sets (or mappings) which agree almost everywhere. It is easy to see that $T$ does not depend on the choice of filtration $\left(K_{m}\right)_{m=1}^{\infty}$.

Definition 1.2. $T$ is called the $(C, F)$-action of $G$ associated with $\left(C_{n}, F_{n}\right)_{n}$.

We will often use the following simple properties of $(X, \mu, T)$ : for Borel subsets $A, B \subset F_{n}$,

$$
\begin{aligned}
& {[A \cap B]_{n}=[A]_{n} \cap[B]_{n},} \\
& {[A]_{n}=\left[A+C_{n+1}\right]_{n+1}=\bigsqcup_{c \in C_{n+1}}[A+c]_{n+1},} \\
& T_{g}[A]_{n}=[A+g]_{n} \quad \text { if } A+g \subset F_{n}, \\
& \mu\left([A]_{n}\right)=\# C_{n+1} \cdot \mu\left([A+c]_{n+1}\right) \quad \text { for every } c \in C_{n+1}, \\
& \mu\left([A]_{n}\right) \leqslant \frac{\lambda_{G}(A)}{\lambda_{G}\left(F_{n}\right)},
\end{aligned}
$$

where the sign $\sqcup$ means the union of mutually disjoint sets.

Recall that an action $T$ of $G$ on $(X, \mathfrak{B}, \mu)$ is partially rigid if there exists $\delta>0$ with

$$
\liminf _{g \rightarrow \infty} \mu\left(T_{g} B \cap B\right) \geqslant \delta \mu(B) \quad \text { for all } B \in \mathfrak{B} .
$$

It is clear that partial rigidity is incompatible with the mixing. For the $(C, F)$-actions, there is a simple condition that implies the partial rigidity.

Proposition 1.3. If $\liminf _{n \rightarrow \infty} \# C_{n}<\infty$ then $T$ is partially rigid and hence is not mixing.

Proof. Let $n_{i}<n_{2}<\cdots$ be a sequence of indices with $\# C_{n_{1}}=\# C_{n_{2}}=\cdots$. Select $c_{i} \neq c_{i}^{\prime}$ in $C_{i}$ and set $g_{i}:=c_{i}-c_{i}^{\prime}$. Then $g_{i} \notin F_{n_{i}}-F_{n_{i}}$ by (1.4). On the other hand, it follows from (1.1) that $\bigcup_{i=1}^{\infty}\left(F_{n_{i}}-F_{n_{i}}\right)=G$. Hence $g_{i} \rightarrow \infty$ as $i \rightarrow \infty$. Take a cylinder $B \in \mathfrak{B}$. We can represent it eventually (i.e., for all large enough $i$ ) as $B=\left[B_{i}\right]_{n_{i}}$, where $B_{i}$ is a Borel subset of $F_{n_{i}}$. If follows from (1.7)-(1.10) that

$$
\begin{aligned}
\mu\left(T_{g_{i}} B \cap B\right) & =\mu\left(T_{g_{i}}\left[B_{i-1}+C_{n_{i}}\right]_{n_{i}} \cap\left[B_{i-1}+C_{n_{i}}\right]_{n_{i}}\right) \\
& \geqslant \mu\left(T_{g_{i}}\left[B_{i-1}+c_{i}^{\prime}\right]_{n_{i}} \cap\left[B_{i-1}+c_{i}\right]_{n_{i}}\right) \\
& =\mu\left(\left[B_{i-1}+c_{i}\right]_{n_{i}}\right)=\frac{1}{\# C_{n_{i}}} \mu\left(\left[B_{i-1}\right]_{n_{i}-1}\right) \\
& =\frac{1}{\# C_{n_{1}}} \mu(B) .
\end{aligned}
$$

Since the cylinders generate a dense subalgebra in $\mathfrak{B}$, we are done.

Now we isolate a special subfamily of $(C, F)$-actions to show in the sequel that it contains mixing actions.

Let $H$ be a discrete countable group, and let $\phi_{n}, s_{n}$ and $c_{n+1}$ be three mappings from $H$ to $G$ such that $\phi_{n}$ is a homomorphism, $s_{n}(0)=0$ and $c_{n+1}:=\phi_{n}+s_{n}, n \in \mathbb{N}$. Suppose that

$$
\left(H_{n}\right)_{n \geqslant 0} \text { is a Følner sequence in } H, 0 \in H_{n}
$$

and

$$
\phi_{n}(H) \text { is a lattice in } G \text {. }
$$


Now we define $F_{n} \subset G$ to be a Borel fundamental domain for $\phi_{n}(H)$ (i.e., a subset which meets every $\phi_{n}(H)$-coset exactly once) and put $C_{n+1}:=c_{n+1}\left(H_{n}\right), n \geqslant 0$. Assume that (1.1)-(1.4) are all satisfied.

Definition 1.4. We call the corresponding $(C, F)$-action $T$ of $G$ on the probability space $(X, \mathfrak{B}, \mu)$ the action associated with $\left(H_{n}, \phi_{n}, s_{n}, F_{n}\right)_{n}$.

In view of Proposition 1.3, we will always assume that $\lim _{n \rightarrow \infty} \# H_{n}=\infty$.

Notice also that if $s_{n}$ are all trivial, i.e. $s_{n}(h)=0$ for all $h \in H_{n}$ then the action of $G$ associated with $\left(H_{n}, \phi_{n}, s_{n}, F_{n}\right)$ has pure point spectrum with rational eigenvalues only. This simple fact will not be used in this paper. We leave its proof to the reader.

In the statements of our main results here it will be assumed that the mappings $s_{n}$ are polynomials of degree $>1$.

Definition 1.5. [16] For any $h \in H$, the $h$-derivative of $s$ is a mapping $\partial_{h} s: H \rightarrow G$ given by $\partial_{h} s(k)=s(k+h)-s(k)$. Let $d$ be a non-negative integer. Then $s$ is called a polynomial of degree $\leqslant d$ if for any $h_{1}, \ldots, h_{d+1} \in H \backslash\{0\}$, we have $\partial_{h_{1}} \cdots \partial_{h_{d+1}} s=0$. The minimal $d$ with this property is called the degree of $s$.

It is easy to see that every polynomial of degree 0 is constant. As was shown in [16], a polynomial of degree one is a non-constant affine mapping (i.e., a homomorphism plus a constant). A polynomial from $\mathbb{Z}^{d}$ to $\mathbb{R}^{l}$ is an $l$-tuple of usual polynomials in $d$ variables with real coefficients. A polynomial from $\mathbb{Z}^{d}$ to $\mathbb{Z}^{l}$ is an $l$-tuple $\left(p_{1}, \ldots, p_{l}\right)$ of usual polynomials in $d$ variables with rational coefficients such that $p_{i}\left(\mathbb{Z}^{d}\right) \subset \mathbb{Z}$ for all $i=1, \ldots, l$.

Remark 1.6. Here we are going to explain how the $(C, F)$-construction for $\mathbb{Z}$-actions is related to the classical cuttingand-stacking construction. Recall that the latter one defines ergodic measure-preserving transformations on intervals in $\mathbb{R}$ (or on $[a,+\infty)$ ) furnished with Lebesgue measure via an inductive procedure. A column is an ordered collection of intervals, called levels, of the same length. The number of levels is called the height of the column. The associated column mapping is defined by translation of each level to the level above it (i.e., next in the order). Hence the column mapping is defined from all but the top level onto all but the bottom level. Suppose now that we are given a sequence $\left(r_{n}\right)_{n=1}^{\infty}$ of positive integers and a sequence of arrays of non-negative integers $\left(\sigma_{n}(j), j=0,1, \ldots, r_{n}-1\right)_{n=1}^{\infty}$. Then we define inductively a sequence of columns as follows. Let the initial column $Y_{0}$ consists of one level of length 1 . Suppose that on the $n$-th step we have a column $Y_{n}$ consisting of levels $I(i, n), 0 \leqslant i<a_{n}$. Cut every $I(i, n)$ into $r_{n}$ sublevels $I_{k}(i, n), 0 \leqslant k<r_{n}$, numbered from left to right. Then we obtain $r_{n}$ subcolumns $Y_{n, k}:=\left\{I_{k}(i, n) \mid i=\right.$ $\left.0, \ldots, a_{n}-1\right\}, 0 \leqslant k<r_{n}$, of $Y_{n}$ of the same height. Now place $\sigma_{n}(k)$ spacers (i.e., the intervals of the same length as $\left.I_{k}(i, n)\right)$ above $Y_{n, k}$ and stack the resulting subcolumns with spacers right to the top of left. This yields a new column $Y_{n+1}$ of height $a_{n+1}=a_{n} r_{n}+\sum_{k=0}^{r_{n}-1} \sigma_{n}(k)$ and a natural inclusion of $Y_{n}$ into $Y_{n+1}$. Notice that the associated $(n+1)$ column mapping restricted to $Y_{n}$ coincides with the $n$-th column mapping. Hence the associated sequence of column mappings approaches a transformation defined on all but a measure zero subset of the union of the initial level and the spacers added at each column. It is easy to see that this transformation corresponds exactly to the $(C, F)$-action of $\mathbb{Z}$ associated with $\left(H_{n}, \phi_{n}, s_{n}, F_{n}\right)_{n}$ if we put $H_{n}:=\left\{0,1, \ldots, r_{n}-1\right\}, \phi_{n}(t):=a_{n} t, s_{n}(t):=\sum_{k=0}^{t} \sigma_{n}(k)$ and $F_{n}=\left\{0,1, \ldots, a_{n}-1\right\}$. If we set $\sigma_{n}(k)=k$ for all $0 \leqslant k<r_{n}$ and $n \in \mathbb{N}$ then the corresponding cutting-and-stacking transformation is called a staircase. If, moreover, $r_{n}=n$ for all $n \in \mathbb{N}$, we obtain the classical staircase which is finite measure-preserving. In case the sequence $\left(\sigma_{n}\right)_{n=1}^{\infty}$ consists of polynomials, the corresponding transformations are called polynomial staircases [5].

\section{Uniformly mixing sequences}

For the remaining of the paper $(X, \mathfrak{B}, \mu, T)$ will stand for the $(C, F)$-action of $G$ associated to a sequence $\left(H_{n}, \phi_{n}, s_{n}, F_{n}\right)_{n}$.

In this section we prove a fundamental Lemma 2.2 and use it to show that some special sequences in $G$ are uniformly mixing. As an auxiliary result for that we exhibit a sufficient condition for the total ergodicity of $T$. A connection between the uniform mixing and total ergodicity is established in Corollary 2.6. 
Definition 2.1. A sequence $\left(g_{n}\right)_{n=1}^{\infty}$ of elements from $G$ is called uniformly mixing for $T$ if

$$
\sup _{A \subset F_{n}}\left|\mu\left(T_{g_{n}}[A]_{n} \cap B\right)-\mu\left([A]_{n}\right) \mu(B)\right| \rightarrow 0 \quad \text { as } n \rightarrow \infty
$$

for every subset $B \in \mathfrak{B}$.

It is easy to see that if a sequence is uniformly mixing then it is mixing.

Notice that a somewhat different definition for uniform mixing was given in [5] in case $G=\mathbb{Z}$. To state it precisely we assume that the sequence $\left(H_{n}, \phi_{n}, s_{n}, F_{n}\right)_{n}$ is chosen as described in Remark 1.6. Then a sequence of positive integers $\left(g_{n}\right)_{n=1}^{\infty}$ was called uniformly mixing in [5] if

$$
\sum_{f \in F_{p_{n}}}\left|\mu\left(T_{g_{n}}[f]_{p_{n}} \cap B\right)-\mu\left([f]_{p_{n}}\right) \mu(B)\right| \rightarrow 0
$$

for every subset $B \in \mathfrak{B}$, where $p_{n}$ is the unique positive integer such that $a_{p_{n}} \leqslant g_{n}<a_{p_{n}+1}$. We only observe that this implies the uniform mixing in the sense of Definition 2.1 if $p_{n} \geqslant n$ for all $n$.

Let $L$ be a finite set and $a: L \rightarrow G$ a mapping. We define a linear operator $\mathcal{M}_{a, L}$ in $L^{2}(X, \mu)$ by setting

$$
\mathcal{M}_{a, L}(f):=\frac{1}{\# L} \sum_{l \in L} f \circ T_{a(l)} .
$$

Let $P_{0}$ stand for the projection onto the subspace of constant functions, i.e. $P_{0}(f)=\int_{X} f \mathrm{~d} \mu$. The inner product in $L^{2}(X, \mu)$ will be denoted by $\langle\cdot, \cdot\rangle$.

Fix a sequence $\left(h_{n}\right)_{n \geqslant 1}$ of elements from $H$. For brevity, we will denote $\partial_{h_{n}} s_{n}$ by $s_{n}^{\prime}, n \in \mathbb{N}$.

Lemma 2.2. If the following conditions are satisfied

$$
\begin{aligned}
& \frac{\# H_{n}\left(h_{n}\right)}{\# H_{n}} \rightarrow 1, \\
& \frac{1}{\# H_{n}} \sum_{h \in H_{n}\left(h_{n}\right)} \frac{\lambda_{G}\left(F_{n} \backslash F_{n}\left(s_{n}^{\prime}(h)\right)\right)}{\lambda_{G}\left(F_{n}\right)} \rightarrow 0
\end{aligned}
$$

then for all $n$-cylinders $A, B \subset X$, we have

$$
\begin{aligned}
\mu\left(T_{\phi_{n}\left(h_{n}\right)} A \cap B\right) & =\frac{1}{\# H_{n}} \sum_{h \in H_{n}\left(h_{n}\right)} \mu\left(A \cap T_{s_{n}^{\prime}(h)} B\right)+\bar{o}(1) \\
& =\left\langle\chi_{A}, \mathcal{M}_{s_{n}^{\prime}, H_{n}\left(h_{n}\right)}\left(\chi_{B}\right)\right\rangle+\bar{o}(1),
\end{aligned}
$$

where $\chi_{A}$ and $\chi_{B}$ are the indicators of $A$ and $B$ respectively and $\bar{o}(1)$ denotes a sequence that tends to 0 and that does not depend on $A$ and $B$. The same formula holds as well for

(i) an arbitrary subset $B \in \mathfrak{B}$ and $A$ as above with $\bar{o}(1)$ depending on $B$ only and

(ii) arbitrary subsets $A, B \in \mathfrak{B}$ with $\bar{o}(1)$ depending on both $A$ and $B$.

Proof. Let $A_{n}$ and $B_{n}$ be the Borel subsets of $F_{n}$ such that $A=\left[A_{n}\right]_{n}$ and $B=\left[B_{n}\right]_{n}$. For $h \in H_{n}\left(h_{n}\right)$, we put $A_{n, h}:=A_{n} \cap F_{n}\left(-s_{n}^{\prime}(h)\right)$. Then

$$
A_{n, h}-s_{n}^{\prime}(h) \subset F_{n} .
$$

We also make a simple but important observation that

$$
\begin{aligned}
\phi_{n}\left(h_{n}\right)+c_{n+1}(h) & =\phi_{n}\left(h_{n}\right)+\phi_{n}(h)+s_{n}(h) \\
& =\phi_{n}\left(h+h_{n}\right)+s_{n}\left(h+h_{n}\right)-s_{n}^{\prime}(h) \\
& =c_{n+1}\left(h+h_{n}\right)-s_{n}^{\prime}(h) .
\end{aligned}
$$

It follows from (1.8), (1.9), (2.4), (1.3), (2.5) and (1.10) that 


$$
\begin{aligned}
\mu\left(T_{\phi_{n}\left(h_{n}\right)} A \cap B\right)= & \sum_{h \in H_{n}} \mu\left(T_{\phi_{n}\left(h_{n}\right)}\left[A_{n}+c_{n+1}(h)\right]_{n+1} \cap\left[B_{n}\right]_{n}\right) \\
= & \sum_{h \in H_{n}\left(h_{n}\right)}\left(\mu\left(T_{\phi_{n}\left(h_{n}\right)}\left[A_{n, h}+c_{n+1}(h)\right]_{n+1} \cap\left[B_{n}\right]_{n}\right)\right. \\
& \left. \pm \mu\left(\left[\left(A_{n} \backslash A_{n, h}\right)+c_{n+1}(h)\right]_{n+1}\right)\right) \pm \sum_{h \in H_{n} \backslash H_{n}\left(h_{n}\right)} \mu\left(\left[F_{n}+c_{n+1}(h)\right]_{n+1}\right) \\
= & \sum_{h \in H_{n}\left(h_{n}\right)}\left(\mu\left(\left[A_{n, h}-s_{n}^{\prime}(h)+c_{n+1}\left(h+h_{n}\right)\right]_{n+1} \cap\left[B_{n}\right]_{n}\right) \pm \frac{1}{\# H_{n}} \mu\left(\left[A_{n} \backslash A_{n, h}\right]_{n}\right)\right) \\
& \pm\left(1-\frac{\# H_{n}\left(h_{n}\right)}{\# H_{n}} r\right) .
\end{aligned}
$$

Notice that for all $c \in C_{n+1}$ and $h \in H_{n}\left(h_{n}\right)$, we have by (1.7), (1.8) and (1.11),

$$
\left[A_{n, h}-s_{n}^{\prime}(h)+c\right]_{n+1} \cap\left[B_{n}\right]_{n}=\left[\left(\left(A_{n, h}-s_{n}^{\prime}(h)\right) \cap B_{n}\right)+c\right]_{n+1}
$$

and

$$
\mu\left(\left[\left(A_{n} \backslash A_{n, h}\right)\right]_{n}\right) \leqslant \frac{\lambda_{G}\left(A_{n} \backslash A_{n, h}\right)}{\lambda_{G}\left(F_{n}\right)} \leqslant \frac{\lambda_{G}\left(F_{n} \backslash F_{n}\left(s_{n}^{\prime}(h)\right)\right)}{\lambda_{G}\left(F_{n}\right)} .
$$

Hence it follows from (1.10), (2.2), (2.3), (2.6) and (2.7) that

$$
\mu\left(T_{\phi_{n}\left(h_{n}\right)} A \cap B\right)=\frac{1}{\# H_{n}} \sum_{h \in H_{n}\left(h_{n}\right)} \mu\left(\left[\left(A_{n, h}-s_{n}^{\prime}(h)\right) \cap B_{n}\right]_{n}\right)+\bar{o}(1) .
$$

Applying (1.7), (1.9) and (2.4) we obtain

$$
\begin{aligned}
\mu\left(T_{\phi_{n}\left(h_{n}\right)} A \cap B\right) & =\frac{1}{\# H_{n}} \sum_{h \in H_{n}\left(h_{n}\right)} \mu\left(T_{-s_{n}^{\prime}(h)}\left[A_{n, h}\right]_{n} \cap\left[B_{n}\right]_{n}\right)+\bar{o}(1) \\
& =\frac{1}{\# H_{n}} \sum_{h \in H_{n}\left(h_{n}\right)}\left(\mu\left(A \cap T_{s_{n}^{\prime}(h)} B\right) \pm \mu\left(\left[A_{n} \backslash A_{n, h}\right]_{n}\right)\right)+\bar{o}(1) .
\end{aligned}
$$

It remains to make use of (2.7), (2.3) and (2.2).

The final claim of Lemma 2.2 follows from the fact that the cylinders generate a dense subalgebra in $\mathfrak{B}$.

Corollary 2.3. Let (2.2) and (2.3) hold. Then the following are satisfied:

(i) The sequence $\left(\phi_{n}\left(h_{n}\right)\right)_{n=1}^{\infty}$ is mixing for $T$ if and only if $\mathcal{M}_{s_{n}^{\prime}, H_{n}\left(h_{n}\right)} \rightarrow P_{0}$ in the weak operator topology.

(ii) If $\mathcal{M}_{s_{n}^{\prime}, H_{n}\left(h_{n}\right)} \rightarrow P_{0}$ in the strong operator topology then $\left(\phi_{n}\left(h_{n}\right)\right)_{n=1}^{\infty}$ is uniformly mixing.

We now examine when $T$ is totally ergodic. Recall some standard definitions.

\section{Definition 2.4.}

(i) Given a subset $B \in \mathfrak{B}$, we denote by $G_{B}$ the stabilizer of $B$, i.e., $G_{B}:=\left\{g \in G \mid T_{g} B=B\right\}$.

(ii) $T$ is called totally ergodic if for any co-compact subgroup $K \subset G$, the action $\left(T_{g}\right)_{g \in K}$ is ergodic.

(iii) $T$ is called weakly mixing if the diagonal action $\left(T_{g} \times T_{g}\right)_{g \in G}$ of $G$ is ergodic. Equivalently, if there exist a function $f \in L^{2}(X, \mu)$ and a continuous character $\chi$ of $G$ such that $f \circ T_{g}=\chi(g) f$ a.e. then $f$ is constant.

It is easy to see that $T$ is totally ergodic if and only if the stabilizer of any subset $B \in \mathfrak{B}$ with $0<\mu(B)<1$ is not co-compact. Moreover, if an action is weakly mixing then it is totally ergodic. The converse is true for $G=\mathbb{R}$ but it does not hold for general groups. 
Proposition 2.5. Let (2.2) and (2.3) hold. Let $K$ be a co-compact subgroup of $G$ and $\pi: G \rightarrow G / K$ stand for the corresponding quotient map. Denote by $\kappa_{n}$ the image of the equidistributed probability on $H_{n}\left(h_{n}\right)$ under the mapping $\left(\pi \circ s_{n}^{\prime}\right)_{*}, n \in \mathbb{N}$. If $\kappa_{n}$ does not $*$-weakly converge to a Dirac $\delta$-measure on $G / K$ then $K$ is not the stabilizer of any measurable subset $B \in \mathfrak{B}$ with $0<\mu(B)<1$.

Proof. Suppose that the contrary holds, i.e., there exists $B \in \mathfrak{B}$ with $0<\mu(B)<1$ and $K=G_{B}$. Then the quotient compact group $G / K$ acts naturally on the sub- $\sigma$-algebra $\mathcal{F}$ of $\left(T_{g}\right)_{g \in K}$-invariant subsets. Denote this action by $\widehat{T}$. Then $\widehat{T}_{\pi(g)} A:=T_{g} A$ for all $g \in G$ and $A \in \mathfrak{F}$. It is clear that $\widehat{T}$ is free. We set $a_{n}:=\pi\left(\phi_{n}\left(h_{n}\right)\right)$. Passing to a subsequence, if necessary, we may assume without loss of generality that $a_{n}$ converges to some $a \in G$. Then $\mu\left(\widehat{T}_{a_{n}} B \Delta \widehat{T}_{a} B\right) \rightarrow 0$ as $n \rightarrow \infty$. Hence

$$
\mu(B)=\mu\left(T_{\phi_{n}\left(h_{n}\right)} B\right)=\mu\left(T_{\phi_{n}\left(h_{n}\right)} B \cap \widehat{T}_{a_{n}} B\right)=\mu\left(T_{\phi_{n}(h)} B \cap \widehat{T}_{a} B\right)+\bar{o}(1) .
$$

We then deduce from this formula and Lemma 2.2(ii) that

$$
\begin{aligned}
\mu(B) & =\frac{1}{\# H_{n}} \sum_{h \in H_{n}\left(h_{n}\right)} \mu\left(B \cap T_{s_{n}^{\prime}(h)} \widehat{T}_{a} B\right)+\bar{o}(1) \\
& =\frac{1}{\# H_{n}\left(h_{n}\right)} \sum_{h \in H_{n}\left(h_{n}\right)} \mu\left(\widehat{T}_{\pi\left(s_{n}^{\prime}(h)\right)+a} B \cap B\right)+\bar{o}(1) \\
& =\int_{G / K} \mu\left(\widehat{T}_{a+b} B \cap B\right) \mathrm{d} \kappa_{n}(b)+\bar{o}(1) .
\end{aligned}
$$

Since $G / K$ is compact, we may assume (passing to a subsequence, if necessary) that $\kappa_{n}$ converges $*$-weakly to a probability $\kappa$ which is not a Dirac $\delta$-measure by the condition of the proposition. Hence passing to a limit in (2.8) we obtain

$$
\mu(B)=\int_{G / H} \mu\left(\widehat{T}_{a+b} B \cap B\right) \mathrm{d} \kappa(b)
$$

Hence $\mu(B)=\mu\left(\widehat{T}_{a+b} B \cap B\right)$, i.e., $B=\widehat{T}_{a+b} B$, for $\kappa$-a.a. $b \in G / K$. Since $\widehat{T}$ is free, we deduce that Supp $\kappa=\{-a\}$. Hence $\operatorname{Supp} \kappa$ is a singleton, a contradiction.

Now we are interested in the following particular case. There exist a non-zero $k \in H$ and a polynomial $s: H \rightarrow G$ of degree 2 such that $h_{n}=k$ and $s_{n}=s$ for all $n \in \mathbb{N}$. Then, of course, (2.2) is satisfied. Moreover, for any non-zero $t \in H$, we have

$$
\partial_{t} s(h)=\psi_{t}(h)+a_{t}, \quad \text { at all } h \in H
$$

for some non-trivial homomorphism $\psi_{t}: H \rightarrow G$ and an element $a_{t} \in G$ (see the text following Definition 1.5). Hence $s_{n}^{\prime}(h)=\psi_{k}(h)+a_{k}$ for all $h \in H$ and $n \in \mathbb{N}$.

Corollary 2.6. Let the above assumptions and (2.3) hold. Then the following are satisfied:

(i) If the action $\left(T_{\psi_{k}(h)}\right)_{h \in H}$ is ergodic then the sequence $\left(\phi_{n}(k)\right)_{n=1}^{\infty}$ is uniformly mixing.

(ii) If the subgroup generated by $\bigcup_{t \in H} \psi_{t}(H)$ is dense in $G$ then $T$ is totally ergodic.

(iii) If the subgroup generated by $\bigcup_{t \in H} \psi_{t}(H)$ is dense in $G$ and $\psi_{k}(H)$ is a lattice in $G$ then the sequence $\left(\phi_{n}(k)\right)_{n=1}^{\infty}$ is uniformly mixing.

Proof. (i) follows from Corollary 2.3 and the von Neumann mean ergodic theorem for $\left(T_{\psi_{k}(h)}\right)_{h \in H}$.

(ii) Suppose that the contrary holds, i.e., there exists a co-compact subgroup $K \subset G$ and a subset $B \subset X$ such that $0<\mu(B)<1$ and $K=G_{B}$. Fix $t \in H, t \neq 0$. Then $\kappa_{n}$ is the translation of $\left(\pi \circ \psi_{t}\right)_{*} v_{n}$ by $\pi\left(a_{t}\right)$, where $v_{n}$ stands for the equidistribution on $H_{n}(t), n \in \mathbb{N}$. Denote by $G_{t}$ the closure of the subgroup $\left(\pi \circ \psi_{t}\right)(H)$ in $G / K$. Since $H_{n}(t)$ is a Følner sequence in $H$ and $\pi \circ \psi_{t}: H \rightarrow G / K$ is a homomorphism, it is easy to verify that $\left(\pi \circ \psi_{t}\right)_{*} \nu_{n}$ converges 
*-weakly to the Haar measure $\lambda_{G_{t}}$ which is considered now as a measure on $G / K$ supported on $G_{t}$. It follows from Proposition 2.5 that Supp $\lambda_{G_{t}}$ is a singleton, i.e. $G_{t}=K$. However we deduce from the condition of the corollary that the subgroup generated by $\bigcup_{t \in H} G_{t}$ is dense in $G / K$. Hence $K=G$ which contradicts to the ergodicity of $T$.

(iii) follows directly from (i) and (ii).

Remark 2.7. We will also need the following slight extension of Corollary 2.6 which is proved in a similar way. Assume that (2.3) holds for a constant sequence $h_{n}=k \neq 0$. Let $S$ be a finite family of polynomials from $H$ to $G$ of degree 2. For all $s \in S$ and $t \in H$, we then have

$$
\partial_{t} s(h)=\psi_{t}^{s}(h)+a_{t}^{s}, \quad h \in H,
$$

where $\psi_{t}^{s}: H \rightarrow G$ is a homomorphism and $a_{t}^{s}$ is an element of $G$. Let $\left(s_{n}\right)_{n=1}^{\infty}$ be a sequence of elements of $S$ such that every element of $S$ occurs in $\left(s_{n}\right)_{n=1}^{\infty}$ infinitely many times. Denote by $T$ the $(C, F)$-action associated with $\left(H_{n}, \phi_{n}, s_{n}, F_{n}\right)_{n}$. Then the following are satisfied.

(i) If the action $\left(T_{\psi_{k}^{s}(h)}\right)_{h \in H}$ is ergodic for any $s \in S$ then the sequence $\left(\phi_{n}(k)\right)_{n=1}^{\infty}$ is uniformly mixing.

(ii) If the subgroup generated by $\bigcup_{s \in S} \bigcup_{t \in H} \psi_{t}^{s}(H)$ is dense in $G$ then $T$ is totally ergodic.

(iii) If the subgroup generated by $\bigcup_{s \in S} \bigcup_{t \in H} \psi_{t}^{s}(H)$ is dense in $G$ and $\psi_{k}^{s}(H)$ is a lattice in $G$ for all $s \in S$ then $\left(\phi_{n}(k)\right)_{n=1}^{\infty}$ is uniformly mixing.

Now we return to the general case and prove two lemmas.

Lemma 2.8. Suppose that there exists a finite subset $Q \subset H$ such that

$$
F_{n}+F_{n} \subset \phi_{n}(Q)+F_{n} \quad \text { for all } n \in \mathbb{N} \text {. }
$$

Let $g_{n}=\phi_{n}(k)+f_{n}$ for some $k \in H$ and $f_{n} \in F_{n}, n \in \mathbb{N}$. If the sequence $\left(\phi_{n}(t)\right)_{n=1}^{\infty}$ is uniformly mixing for every $t \in k+Q$ then so is the sequence $\left(g_{n}\right)_{n=1}^{\infty}$.

Proof. Since $f_{n}+F_{n} \subset \phi_{n}(Q)+F_{n}$ and $F_{n}$ is a fundamental domain for $\phi_{n}(H)$, a finite partition of $F_{n}$ is well defined as follows: $F_{n}=\bigsqcup_{q \in Q} F_{n}\left(f_{n}-\phi_{n}(q)\right)$. Now let $A$ be an $n$-cylinder in $X$ and $A=\left[A_{n}\right]_{n}$ for some Borel subset $A_{n} \subset F_{n}$. Consider the induced partition $\bigsqcup_{q \in Q} A_{n, q}$ of $A_{n}$, where $A_{n, q}:=A_{n} \cap F\left(f_{n}-\phi_{n}(q)\right)$. Then by (1.9),

$$
T_{g_{n}}\left[A_{n, q}\right]_{n}=T_{\phi_{n}(k+q)} T_{f_{n}-\phi_{n}(q)}\left[A_{n, q}\right]_{n}=T_{\phi_{n}(k+q)}\left[f_{n}-\phi_{n}(q)+A_{q}\right]_{n} .
$$

Hence for any subset $B \in \mathfrak{B}$,

$$
\mu\left(T_{g_{n}} A \cap B\right)-\mu(A) \mu(B)=\sum_{q \in Q}\left(\mu\left(T_{\phi_{n}(k+q)}\left[A_{n, q}\right]_{n} \cap B\right)-\mu\left(\left[A_{n, q}\right]_{n} \cap B\right)\right) .
$$

It remains to use the uniform mixing of $\left(\phi_{n}(t)\right)_{n=1}^{\infty}, t \in k+Q$.

Suppose that—in addition to (2.9)—-there is a finite subset $Q^{\bullet} \subset H$ such that

$$
F_{n}-F_{n} \subset \phi_{n}\left(Q^{\bullet}\right)+F_{n} \text { for all } n \in \mathbb{N} .
$$

We will assume in the following that $Q^{\bullet}$ is the minimal subset with this property. It is clear that $0 \in Q^{\bullet}$. We set $F_{n}^{\bullet}:=\phi_{n}\left(Q^{\bullet}\right)+F_{n}$. Then there exists a finite subset $Q_{+} \subset H$ such that

$$
\left(F_{n}^{\bullet}+F_{n}^{\bullet}\right) \backslash F_{n}^{\bullet} \subset \phi_{n}\left(Q_{+}\right)+F_{n} \quad \text { for all } n \in \mathbb{N} .
$$

Again we will assume that $Q_{+}$is the minimal subset with this property. It is clear that $Q^{\bullet} \cap Q_{+}=\emptyset$.

Lemma 2.9. Let (2.9) and (2.10) hold and let for any $t \in H \backslash\{0\}$, the sequence $\left(\phi_{n}(t)\right)_{n=1}^{\infty}$ be uniformly mixing. Take $g_{n} \in\left(F_{n}^{\bullet}+F_{n}^{\bullet}\right) \backslash F_{n}^{\bullet}$ for all $n \in \mathbb{N}$. If for an integer $l>0$, we have

$$
-l Q_{+} \cap(\underbrace{Q+\cdots+Q}_{l \text { times }})=\emptyset
$$

then the sequence $\left(\lg _{n}\right)_{n=1}^{\infty}$ is uniformly mixing. 
Proof. Since $Q_{+}$is finite, we can partition the sequence $g_{n}$ into finitely many subsequences of the form $\phi_{n}(q)+f_{n}$ for some $f_{n} \in F_{n}, n \in \mathbb{N}$, and a fixed $q \in Q_{+}$. Therefore it is enough to assume that $g_{n}$ itself enjoys this property. Then condition (2.9) applied $l-1$ times yields

$$
l g_{n}=\phi_{n}(l q)+\underbrace{f_{n}+\cdots+f_{n}}_{l \text { times }}=\phi_{n}(l q)+\phi_{n}\left(q_{1}+\cdots+q_{l-1}\right)+\tilde{f}_{n}
$$

for some $q_{1}, \ldots, q_{l-1} \in Q$ and $\tilde{f}_{n} \in F_{n}$. Since $0 \notin l q+q_{1}+\cdots+q_{l-1}+Q$ by the condition of the lemma, we may apply Lemma 2.8 to complete the proof.

\section{Restricted growth condition}

In this section we introduce a restricted growth condition for the $(C, F)$-actions specified in Section 2. It is an analogue of a concept considered in [5] for $G=\mathbb{Z}$. The concept, in turn, is a counterpart of a sufficient condition introduced in [1] for a staircase to be mixing. The main result here is Theorem 3.5, which provides a sufficient condition for mixing in the class of $(C, F)$-actions with restricted growth. We conclude the section with some technical statements needed to verify this condition for some special $\mathbb{R}^{d_{1}} \times \mathbb{Z}^{d_{2}}$-actions that will be constructed in the next section.

Definition 3.1. We say that $T$ satisfies the restricted growth condition if (2.3) holds for any sequence $h_{n} \in H$ such that there exists $\lim _{n \rightarrow \infty} \# H_{n}\left(h_{n}\right) / \# H_{n}>0$.

Definition 3.2. Given two sequences $A_{n}, B_{n} \subset G$, we write $A_{n} \sim_{n} B_{n}$ if

$$
\frac{\lambda_{G}\left(A_{n} \Delta B_{n}\right)}{\lambda_{G}\left(F_{n}\right)} \rightarrow 0 \quad \text { as } n \rightarrow \infty
$$

It follows from (1.11) that $\mu\left(\left[A_{n}\right]_{n} \Delta\left[B_{n}\right]_{n}\right) \rightarrow 0$ whenever $A_{n} \sim_{n} B_{n}$ and $A_{n}, B_{n} \subset F_{n}$.

Lemma 3.3. Suppose that (2.9) and the following hold

$$
\frac{1}{\# H_{n}} \sum_{h \in H_{n}} \frac{\lambda_{G}\left(F_{n} \backslash F_{n}\left(s_{n}(h)\right)\right)}{\lambda_{G}\left(F_{n}\right)} \rightarrow 0 \quad \text { as } n \rightarrow \infty .
$$

Let $g_{n}:=\phi_{n}\left(h_{n}\right)+f_{n}$ for some $h_{n} \in H$ and $f_{n} \in F_{n}, n \in \mathbb{N}$. Then

$$
F_{n+1}\left(g_{n}\right) \sim_{n+1} \bigsqcup_{h \in H_{n}\left(h_{n}\right)}\left(c_{n+1}(h)+F_{n}\right) .
$$

Proof. Since

$$
\begin{aligned}
& \lambda_{G}\left(F_{n+1} \Delta \bigsqcup_{h \in H_{n}}\left(\phi_{n}(h)+F_{n}\right) r\right) \leqslant \lambda_{G}\left(F_{n+1} \backslash\left(F_{n}+C_{n+1}\right)\right)+\sum_{h \in H_{n}} \lambda_{G}\left(\left(c_{n+1}(h)+F_{n}\right) \Delta\left(\phi_{n}(h)+F_{n}\right)\right) \\
& \quad=\lambda_{G}\left(F_{n+1} \backslash\left(F_{n}+C_{n+1}\right)\right)+\sum_{h \in H_{n}} \lambda_{G}\left(\left(s_{n}(h)+F_{n}\right) \Delta F_{n}\right),
\end{aligned}
$$

it follows from (3.1) and (1.6) that $F_{n+1} \sim_{n+1}\left(\phi_{n}\left(H_{n}\right)+F_{n}\right)$. For $q \in Q$, we set $F_{n, q}:=F_{n}\left(f_{n}-\phi_{n}(q)\right)$ and $F_{n, q}^{\prime}:=$ $F_{n}\left(-f_{n}+\phi_{n}(q)\right)$. Then (2.9) yields

$$
F_{n}=\bigsqcup_{q \in Q} F_{n, q}=\bigsqcup_{q \in Q} F_{n, q}^{\prime} .
$$

It follows that

$$
F_{n+1}-g_{n} \sim_{n+1} \bigsqcup_{h \in H_{n}} \bigsqcup_{q \in Q}\left(\phi_{n}(h)-\phi_{n}\left(h_{n}\right)-f_{n}+F_{n, q}^{\prime}\right)=\bigsqcup_{q \in Q} \bigsqcup_{h \in H_{n}}\left(\phi_{n}\left(h-h_{n}-q\right)+F_{n, q}\right) .
$$


Therefore

$$
F_{n+1} \cap\left(F_{n+1}-g_{n}\right) \sim_{n+1} \bigsqcup_{q \in Q} \bigsqcup_{h \in H_{n}\left(h_{n}+q\right)}\left(\phi_{n}(h)+F_{n, q}\right) .
$$

Next, since $\lambda_{G}\left(F_{n+1}\right) \geqslant \# H_{n} \lambda_{G}\left(F_{n}\right)$ and

$$
\begin{aligned}
\frac{\lambda_{G}\left(\bigsqcup_{q \in Q} \bigsqcup_{h \in H_{n}\left(h_{n}+q\right) \Delta H_{n}\left(h_{n}\right)}\left(\phi_{n}(h)+F_{n, q}\right)\right)}{\lambda_{G}\left(F_{n+1}\right)} & \leqslant \frac{\sum_{q \in Q} \#\left(H_{n}\left(h_{n}+q\right) \Delta H_{n}\left(h_{n}\right)\right) \lambda_{G}\left(F_{n, q}\right)}{\# H_{n} \lambda_{G}\left(F_{n}\right)} \\
& \leqslant \sum_{q \in Q} \frac{\#\left(H_{n}\left(h_{n}+q\right) \Delta H_{n}\left(h_{n}\right)\right)}{\# H_{n}} \rightarrow 0,
\end{aligned}
$$

it follows from (3.3) and (3.2) that

$$
F_{n+1}\left(g_{n}\right) \sim_{n+1} \bigsqcup_{q \in Q} \bigsqcup_{h \in H_{n}\left(h_{n}\right)}\left(\phi_{n}(h)+F_{n, q}\right)=\bigsqcup_{h \in H_{n}\left(h_{n}\right)}\left(\phi_{n}(h)+F_{n}\right) .
$$

It remains to use (3.1) once more to conclude that

$$
\bigsqcup_{h \in H_{n}\left(h_{n}\right)}\left(\phi_{n}(h)+F_{n}\right) \sim_{n+1} \bigsqcup_{h \in H_{n}\left(h_{n}\right)}\left(c_{n+1}(h)+F_{n}\right) .
$$

Corollary 3.4. If (2.9) and (3.1) are satisfied then

$$
\left(A+C_{n+1}\right) \cap F_{n+1}\left(g_{n}\right) \sim_{n+1} A+c_{n+1}\left(H_{n}\left(h_{n}\right)\right)
$$

uniformly in $A \subset F_{n}$. Hence

$$
\sup _{A \subset F_{n}} \mu\left(\left([A]_{n} \cap\left[F_{n+1}\left(g_{n}\right)\right]_{n+1}\right) \Delta\left[A+c_{n+1}\left(H_{n}\left(h_{n}\right)\right)\right]_{n+1}\right)=\bar{o}(1) .
$$

Now we are going to prove the main result of the section.

Theorem 3.5. Let $T$ be a $(C, F)$-action associated with $\left(H_{n}, \phi_{n}, s_{n}, F_{n}\right)_{n}$ and satisfying (2.9), (2.10), (3.1) and the restricted growth condition. Let $Q^{\bullet} \supset-Q$. If $\mathcal{M}_{s_{n}^{\prime}, H_{n}\left(h_{n}\right)} \rightarrow P_{0}$ strongly for any sequence $h_{n} \in H \backslash\{0\}$ such that

$$
\liminf _{n \rightarrow \infty} \# H_{n}\left(h_{n}\right) / \# H_{n}>0
$$

then $T$ is mixing. (Here $s_{n}^{\prime}$ stands for the $h_{n}$-derivative of $s_{n}$.)

Proof. Take any sequence $g_{n} \rightarrow \infty$ in $G$. We are going to prove that it contains a mixing subsequence. Since $F_{1}^{\bullet} \subset F_{2}^{\bullet} \subset \cdots$ and $\bigcup_{n=1}^{\infty} F_{n}^{\bullet}=G$, we can find $p_{n}$ such that $g_{n} \in F_{p_{n}+1}^{\bullet} \backslash F_{p_{n}}^{\bullet}$ for all $n \in \mathbb{N}$. It is clear that $p_{n} \rightarrow \infty$ Notice that

$$
F_{n+1}^{\bullet}+F_{n+1} \subset \phi_{n+1}\left(Q^{\bullet}+Q\right)+F_{n+1} .
$$

Then using a similar idea as in the proof of Lemma 2.8, we partition $F_{p_{n}+1}$ as follows

$$
F_{p_{n}+1}=\bigsqcup_{q \in Q^{\bullet}+Q} F_{p_{n}+1}\left(g_{n}-\phi_{p_{n}+1}(q)\right) .
$$

Take two $p_{n}$-cylinders $A, B \subset X$. Let $A_{m}$ and $B_{m}$ be the Borel subsets of $F_{m}$ such that $A=\left[A_{m}\right]_{m}$ and $B=\left[B_{m}\right]_{m}$ for all $m \geqslant p_{n}$. For $q \in Q^{\bullet}+Q$, we put $A_{p_{n}+1, q}:=A_{p_{n}+1} \cap F_{p_{n}+1}\left(g_{n}-\phi_{p_{n}}(q)\right)$. We have now

$$
T_{g_{n}}\left[A_{p_{n}+1, q}\right]_{p_{n}+1}=T_{\phi_{p_{n}+1}(q)}\left[A_{p_{n}+1, q}+g_{n}-\phi_{p_{n}+1}(q)\right]_{p_{n}+1}
$$

and $A_{p_{n}+1}=\bigsqcup_{q \in Q^{\bullet}+Q} A_{p_{n}+1, q}$. For any $q \in Q^{\bullet}+Q$, the constant sequence $h_{n}:=q, n \in \mathbb{N}$ satisfies (2.2). Moreover, it satisfies (2.3) since $T$ has restricted growth (we need this to apply Corollary 2.3 later). By the condition of the 
theorem, $\mathcal{M}_{\partial_{q} s_{n}, H_{n}(q)} \rightarrow P_{0}$ strongly whenever $q \neq 0$. Therefore by Corollary 2.3(ii), the sequence $\left(\phi_{n}(q)\right)_{n=1}^{\infty}$ is uniformly mixing. Then it follows from (3.4) that

$$
\sup _{A \text { is a } p_{n} \text {-cylinder }}\left|\mu\left(T_{g_{n}}\left[A_{p_{n}+1, q}\right]_{p_{n}+1} \cap B\right)-\mu\left(\left[A_{p_{n}+1, q}\right]_{p_{n}+1}\right) \mu(B)\right| \rightarrow 0
$$

for every $q \in\left(Q^{\bullet}+Q\right) \backslash\{0\}$.

It remains to consider the case $q=0$ which is more involved. Let us represent $g_{n}$ as $g_{n}=\phi_{p_{n}}\left(h_{n}\right)+f_{n}$ for some (uniquely determined) $h_{n} \in H \backslash Q^{\bullet}$ and $f_{n} \in F_{p_{n}}$. Since $A_{p_{n}+1,0}=A_{p_{n}+1} \cap F_{p_{n}+1}\left(g_{n}\right)$ and (3.1) holds, it follows from Corollary 3.4 that

$$
\sup _{A \text { in a } p_{n} \text {-cylinder }}\left|\mu\left(\left[A_{p_{n}+1,0}\right]_{p_{n}+1}\right)-\mu(A) \frac{\# H_{p_{n}}\left(h_{n}\right)}{\# H_{p_{n}}}\right|=\bar{o}(1) .
$$

Hence, if $\liminf _{n \rightarrow \infty} \# H_{p_{n}}\left(h_{n}\right) / \# H_{p_{n}}=0$,

$$
\liminf _{n \rightarrow \infty} \sup _{A \text { in a } p_{n} \text {-cylinder }} \mu\left(\left[A_{p_{n}+1,0}\right]_{p_{n}+1}\right)=0 .
$$

Therefore passing to a subsequence in $\left(p_{n}\right)_{n=1}^{\infty}$ we conclude that (3.5) is also true for $q=0$. This implies that the corresponding subsequence of $\left(g_{n}\right)_{n=1}^{\infty}$ is mixing. Thus from now on we may assume that $\liminf _{n \rightarrow \infty} \# H_{p_{n}}\left(h_{n}\right) / \# H_{p_{n}}>0$. It follows from Corollary 3.4 that

$$
\mu\left(T_{\phi_{p_{n}}\left(h_{n}\right)}\left[A_{p_{n}+1,0}\right]_{p_{n}+1} \cap B\right)=\mu\left(T_{\phi_{p_{n}}\left(h_{n}\right)}\left[A_{p_{n}}+c_{p_{n}+1}\left(H_{p_{n}}\left(h_{n}\right)\right)\right]_{p_{n}+1} \cap B\right)+\bar{o}(1),
$$

where $\bar{o}(1)$ does not depend on the $p_{n}$-cylinders $A$ and $B$. Using that together with the restricted growth condition and arguing almost literally as in the proof of Lemma 2.2 we obtain

$$
\begin{aligned}
\mu\left(T_{\phi_{p_{n}}\left(h_{n}\right)}\left[A_{p_{n}+1,0}\right]_{p_{n}+1} \cap B\right) & =\frac{1}{\# H_{p_{n}}} \sum_{h \in H_{p_{n}}\left(h_{n}\right)} \mu\left(T_{-s_{n}^{\prime}(h)}\left[A_{p_{n}}\right]_{p_{n}} \cap B\right)+\bar{o}(1) \\
& =\frac{\# H_{p_{n}}\left(h_{n}\right)}{\# H_{p_{n}}}\left\langle\chi_{A}, \mathcal{M}_{s_{n}^{\prime}, H_{p_{n}}\left(h_{n}\right)}\left(\chi_{B}\right)\right\rangle+\bar{o}(1),
\end{aligned}
$$

where $s_{n}^{\prime}$ is the $h_{p_{n}}$-derivative of $s_{n}$ and $\bar{o}(1)$ does not depend on $A$ and $B$. It is easy to find a sequence $\left(\hat{h}_{n}\right)_{n=1}^{\infty}$ with $\liminf _{n \rightarrow \infty} \# H_{n}\left(\hat{h}_{n}\right) / \# H_{n}>0, \hat{h}_{n} \neq 0$ and $\hat{h}_{p_{n}}=h_{n}$ for all $n \in \mathbb{N}$. Let $\hat{s}_{n}^{\prime}$ denote the $\hat{h}_{n}$-derivative of $s_{n}$. Then $s_{n}^{\prime}=\hat{s}_{p_{n}}^{\prime}$. By the condition of the theorem, $\mathcal{M}_{\hat{s}_{n}^{\prime}, H_{n}\left(\hat{h}_{n}\right)} \rightarrow P_{0}$ strongly. Hence $\mathcal{M}_{s_{n}^{\prime}, H_{p_{n}}\left(h_{n}\right)} \rightarrow P_{0}$ strongly. Therefore we deduce from the above and (3.6) that

$$
\begin{aligned}
\mu\left(T_{\phi_{p_{n}}\left(h_{n}\right)}\left[A_{p_{n}+1,0}\right]_{p_{n}+1} \cap B\right) & =\frac{\# H_{p_{n}}\left(h_{n}\right)}{\# H_{p_{n}}} \cdot \mu(A) \mu(B)+\bar{o}(1) \\
& =\mu\left(\left[A_{n+1,0}\right]_{p_{n}+1}\right) \mu(B)+\bar{o}(1),
\end{aligned}
$$

where $\bar{o}(1)$ does not depend on $A$. To complete the proof, we will use once more the 'partition trick'. Let $A_{p_{n}}^{q}:=$ $A_{p_{n}} \cap F_{p_{n}}\left(f_{n}-\phi_{p_{n}}(q)\right)$ for $q \in Q$. Then in view of (2.9) $A_{p_{n}}=\bigsqcup_{q \in Q} A_{p_{n}}^{q}$ and $f_{n}-\phi_{p_{n}}(q)+A_{p_{n}}^{q} \subset F_{p_{n}}$ for all $q \in Q$. It is easy to see that (3.7) is also true if we replace the sequence $\left(h_{n}\right)_{n \geqslant 1}$ with the sequence $\left(h_{n}+q\right)_{n \geqslant 1}$ for any $q \in Q$. Notice that $h_{n}+q \neq 0$ since $Q^{\bullet} \supset-Q$. Hence applying Corollary 3.4 and (3.7) we obtain

$$
\begin{aligned}
\mu\left(T_{g_{n}}\left[A_{p_{n}+1,0}\right]_{p_{n}+1} \cap B\right) & =\mu\left(T_{g_{n}}\left[A_{p_{n}}+c_{p_{n}+1}\left(H_{p_{n}}\left(h_{n}\right)\right)\right]_{p_{n}+1} \cap B\right)+\bar{o}(1) \\
& =\sum_{q \in Q} \mu\left(T_{\phi_{p_{n}}\left(h_{n}+q\right)}\left[f_{n}-\phi_{p_{n}}(q)+A_{p_{n}}^{q}+c_{p_{n}+1}\left(H_{p_{n}}\left(h_{n}\right)\right)\right]_{p_{n}+1} \cap B\right)+\bar{o}(1) \\
& =\sum_{q \in Q} \mu\left(\left[f_{n}-\phi_{p_{n}}(q)+A_{p_{n}}^{q}+c_{p_{n}+1}\left(H_{p_{n}}\left(h_{n}\right)\right)\right]_{p_{n}+1}\right) \mu(B)+\bar{o}(1) \\
& =\sum_{q \in Q} \mu\left(\left[A_{p_{n}}^{q}+c_{p_{n}+1}\left(H_{p_{n}}\left(h_{n}\right)\right)\right]_{p_{n}+1}\right) \mu(B)+\bar{o}(1) \\
& =\mu\left(\left[A_{p_{n}}+c_{p_{n}+1}\left(H_{p_{n}}\left(h_{n}\right)\right)\right]_{p_{n}+1}\right) \mu(B)+\bar{o}(1) \\
& =\mu\left(\left[A_{p_{n}+1,0}\right]_{p_{n}+1}\right) \mu(B)+\bar{o}(1) .
\end{aligned}
$$


This plus (3.5) imply that the sequence $\left(g_{n}\right)_{n=1}^{\infty}$ is mixing.

The following material will be used in the next section to check the condition on the strong convergence from Theorem 3.5.

Definition 3.6. Let $I, J$ be finite subsets in $H$ and let $\epsilon$ be a non-negative number. We say that $I$ is $\epsilon$-tiled by $J$ if there exists a finite set $F \subset H$ such that the following are satisfied:

(i) $J+F \subset I$,

(ii) $J$ and $F$ are independent and

(iii) $\#(I \backslash(J+F)) \leqslant \epsilon \# I$.

Lemma 3.7. Let I be $\epsilon$-tiled by $J$. Then for any function $f \in L^{2}(X, \mu)$ and any homomorphism $\alpha: H \rightarrow G$,

$$
\left\|\mathcal{M}_{\alpha, I}(f)\right\|_{2} \leqslant\left\|\mathcal{M}_{\alpha, J}(f)\right\|_{2}+\epsilon\|f\|_{2} \text {. }
$$

Proof. Since $T$ preserves $\mu$, it follows that

$$
\left\|\mathcal{M}_{\alpha, h+J}(f)\right\|_{2}=\left\|\mathcal{M}_{\alpha, J}(f) \circ T_{\alpha(h)}\right\|_{2}=\left\|\mathcal{M}_{\alpha, J}(f)\right\|_{2}
$$

for every $h \in H$. Let $F$ be as in Definition 3.6. Then

$$
\begin{aligned}
\left\|\mathcal{M}_{\alpha, I}(f)\right\|_{2} & \leqslant \frac{\# J}{\# I} \sum_{h \in F}\left\|\mathcal{M}_{\alpha, h+J}(f)\right\|_{2}+\frac{\#(I \backslash(J+F))}{\# I}\left\|\mathcal{M}_{\alpha, I \backslash(J+F)}(f)\right\|_{2} \\
& \leqslant\left\|\mathcal{M}_{\alpha, J}(f)\right\|_{2}+\epsilon\|f\|_{2} .
\end{aligned}
$$

Let $h \in H, i, j \in \mathbb{Z}$ and $0 \leqslant i<j$. The subset $\{i h,(i+1) h, \ldots, j h\}$ is called an $h$-interval.

Remark 3.8. Let $V \subset H$ be a finite subset, $I, J$ two $h$-intervals in $H$ and $l \in \mathbb{N}$. If $V$ is tiled (i.e., 0 -tiled) by $I$ then $V$ is also $\frac{l \# J}{\# I}$-tiled by the $l h$-interval $l \cdot J$.

Lemma 3.9. Let $\alpha: H \rightarrow G$ be a homomorphism. Let $\left(h_{n}\right)_{n} \geqslant 1$ be a sequence in $H$ such that for some subset $A \subset X$ and every $l \in \mathbb{N}$, we have $\mu\left(T_{l \alpha}\left(h_{n}\right) A \cap A\right) \rightarrow \mu(A)^{2}$ as $n \rightarrow \infty$. Then for any sequence of $h_{n}$-intervals $I_{n}$ whose cardinality is constant, say $L$, the following inequality holds eventually (i.e., for all large enough $n$ )

$$
\left\|\mathcal{M}_{\alpha, I_{n}}\left(\chi_{A}\right)-\mu(A)\right\|_{2}^{2} \leqslant \frac{2}{L} \text {. }
$$

Proof. Since $T$ preserves $\mu$, without loss of generality we may assume that $I_{n}=\left\{0, h_{n}, \ldots,(L-1) h_{n}\right\}$. Now the inequality follows from the formula

$$
\left\|\frac{1}{L} \sum_{i=0}^{L-1} \chi_{A} \circ T_{i \alpha\left(h_{n}\right)}-\mu(A)\right\|_{2}^{2}=\frac{\mu(A)}{L}+\frac{1}{L^{2}} \sum_{i \neq j}\left(\mu\left(T_{|i-j| \alpha\left(h_{n}\right)} A \cap A\right)-\mu(A)^{2}\right),
$$

which is established by a straightforward calculation.

Lemma 3.10. Let $\alpha: H \rightarrow G$ be a homomorphism, $\left(h_{n}\right)_{n \geqslant 1}$ a sequence in $H$ and $\left(m_{n}\right)_{n} \geqslant 1$ a sequence in $\mathbb{N}$. Let $V_{n}$ be a finite subset of $H$ which is tiled by an $h_{n}$-interval $I_{n}$. If $m_{n} / \# I_{n} \rightarrow 0$ and $\mu\left(T_{l m_{n} \alpha\left(h_{n}\right)} A \cap A\right) \rightarrow \mu(A)^{2}$ as $n \rightarrow \infty$ for any integer $l>0$ then $\mathcal{M}_{\alpha, V_{n}}\left(\chi_{A}\right) \rightarrow \mu(A)$ in $L^{2}(X, \mu)$.

Proof. For an $\epsilon>0$, fix an integer $r>\epsilon^{-1}$. Let $J_{n}$ be a $h_{n}$-interval of cardinality $r$. By Lemma 3.9,

$$
\left\|\mathcal{M}_{\alpha, m_{n} J_{n}}\left(\chi_{A}\right)-\mu(A)\right\|_{2}^{2}<2 \epsilon
$$


eventually in $n$. It follows from Remark 3.8 that $V_{n}$ is $\frac{m_{n} r}{\# I_{n}}$-tiled by the $m_{n} h_{n}$-interval $m_{n} J_{n}$. By Lemma 3.7,

$$
\left\|\mathcal{M}_{\alpha, V_{n}}\left(\chi_{A}\right)-\mu(A)\right\|_{2} \leqslant\left\|\mathcal{M}_{\alpha, m_{n} J_{n}}\left(\chi_{A}\right)-\mu(A)\right\|_{2}+\frac{m_{n} r}{\# I_{n}}<3 \epsilon
$$

eventually in $n$.

The following results will be used while proving mixing of the $(C, F)$-actions whose 'spacer mappings' $s_{n}$ are polynomials of degree $>2$.

Lemma 3.11 (Hilbertian van der Corput trick). Let $\left(v_{h}\right)_{h \in H}$ stand for a bounded family of vectors in a Hilbert space. If for any $k \in H \backslash\{0\}$, we have $\lim _{n \rightarrow \infty} \frac{1}{\# H_{n}} \sum_{h \in H_{n}}\left\langle v_{h+k}, v_{h}\right\rangle=0$ then $\lim _{n \rightarrow \infty}\left\|\frac{1}{\# H_{n}} \sum_{h \in H_{n}} v_{h}\right\|=0$.

Proof. In case $H=\mathbb{Z}$, we refer the reader to [4] for a short proof. Only a slight and obvious modification of that proof is needed to adapt it to the general case.

Corollary 3.12. Given a map $t: H \rightarrow G$, let $\left\langle\mathcal{M}_{\partial_{k} t, H_{n}} \chi_{B}, \chi_{B}\right\rangle \rightarrow \mu(B)^{2}$ for every non-trivial $k \in H$ and all $B \in \mathfrak{B}$. Then $\mathcal{M}_{t, H_{n}} \rightarrow P_{0}$ strongly as operators in $L^{2}(X, \mu)$.

Proof. Fix a subset $B \in \mathfrak{B}$. For any $h \in H$, we set $v_{h}:=\chi_{B} \circ T_{t(h)}-\mu(B) \in L^{2}(X, \mu)$. Then $\left\langle v_{h+k}, v_{h}\right\rangle=$ $\left\langle\chi_{B} \circ T_{\partial_{k} t(h)}, \chi_{B}\right\rangle-\mu(B)^{2}$. It remains to apply Lemma 3.11.

\section{Mixing rank-one actions of $\mathbb{R}^{d_{1}} \times \mathbb{Z}^{d_{2}}$}

In this section we prove the main results of the paper-Theorems 4.9-4.11 and 4.12.

Let $d_{1}$ and $d_{2}$ be non-negative integers such that $d:=d_{1}+d_{2} \neq 0$. We set $G=\mathbb{R}^{d_{1}} \times \mathbb{Z}^{d_{2}}$ and $H=\mathbb{Z}^{d}$. For $g=\left(g_{1}, \ldots, g_{d}\right) \in G$, we let $\|g\|_{\infty}:=\max _{1 \leqslant i \leqslant d}\left|g_{i}\right|$. If $g_{i} \geqslant 0$ for all $i=1, \ldots, d$ we write $g \geqslant 0$. In a similar way we define $\|h\|_{\infty}$ and $h \geqslant 0$ for $h \in H$.

To define a mixing $(C, F)$-action of $G$ we first fix a sequence of positive integers $r_{n}>2$ which goes to infinity as $n \rightarrow \infty$. Some restrictions on its growth will be imposed later. Let $s_{n}^{(1)}: H \rightarrow \mathbb{R}^{d_{1}}$ be a usual polynomial with real coefficients and $s_{n}^{(2)}: H \rightarrow \mathbb{Z}^{d_{2}}$ a usual polynomial with rational coefficients such that $s_{n}^{(1)}(0)=0$ and $s_{n}^{(2)}(0)=0$. Then $s_{n}:=\left(s_{n}^{(1)}, s_{n}^{(2)}\right)$ is a polynomial mapping from $H$ to $G$ in the sense of Definition 1.5. Let $H_{n}:=\{h \geqslant 0 \mid$ $\left.\|h\|_{\infty}<r_{n}\right\}$. We define a sequence of positive reals $\left(a_{n}\right)_{n \geqslant 0}$ recurrently by setting

$$
a_{n+1}:=\text { the integer part of } a_{n} r_{n}+\max _{h \in H_{n}}\left\|s_{n}(h)\right\|_{\infty}
$$

and choosing $a_{0}$ arbitrarily. It is clear that $a_{n+1} \geqslant a_{0} r_{1} \cdots r_{n}$ for all $n \in \mathbb{N}$. Since $r_{n} \rightarrow \infty$, the sequence $\left(a_{n}\right)_{n=1}^{\infty}$ grows faster than any exponent. We finally let

$$
\begin{aligned}
& F_{n}:=\left\{g \geqslant 0 \mid\|g\|_{\infty}<a_{n}\right\}, \\
& \phi_{n}(h):=a_{n} h \quad \text { for } h \in H \text { and } \\
& C_{n+1}:=\left(\phi_{n}+s_{n}\right)\left(H_{n}\right),
\end{aligned}
$$

for $n=0,1, \ldots$ It is easy to see that (1.1)-(1.3), (1.12) and (1.13) are satisfied. In the case $d=1$, the condition (1.4) is satisfied too if $s_{n}$ is non-negative and non-decreasing on $\mathbb{Z}_{+}$, for instance if the coefficients of $s_{n}$ are all non-negative. The situation is more difficult when $d>1$. That is why we introduce the following definition.

Definition 4.1. A mapping $s: H \rightarrow G$ is monotonic if for all non-negative $x, y \in H$ with $\|x-y\|_{\infty}=1$,

$$
\max \left\{\frac{s(x)_{i}-s(y)_{i}}{x_{i}-y_{i}} \mid \text { for all } 1 \leqslant i \leqslant d \text { such that } x_{i} \neq y_{i}\right\} \geqslant 0 .
$$


Example 4.2. Let $s(x)_{i}=\left(\alpha_{i} x_{i}+\gamma_{i}\right)\left(x_{1}+\cdots+x_{d}\right)+\beta_{i} x_{i}^{2}+\delta_{i} x_{i}$ for some reals $\alpha_{i}, \beta_{i}, \gamma_{i}$ and $\delta_{i}$ such that $\alpha_{i}>0$, $\gamma_{i} \geqslant 0, \alpha_{i}+2 \beta_{i} \geqslant 0$ and $\alpha_{i}+\beta_{i}+\delta_{i} \geqslant 0$ for $i=1, \ldots, d$. Suppose that the mapping

$$
H \ni x \mapsto s(x)=\left(s(x)_{1}, \ldots, s(x)_{d}\right)
$$

takes values in $G$. Then it is monotonic. To show this, consider two non-negative elements $x \neq y \in H$ such that $\|x-y\|_{\infty}=1$ and set $z:=y-x$. Without loss of generality we may assume that $z_{1}+\cdots+z_{d} \geqslant 0$. Then there exists a coordinate $j$ such that $z_{j}=1$. By a straightforward calculation,

$$
s(x+z)_{j}-s(x)_{j}=\left(\alpha_{j} x_{j}+\gamma_{j}\right) \sum_{i=1}^{d} z_{i}+\alpha_{j} \cdot \sum_{i \neq j}\left(x_{i}+z_{i}\right)+\left(\alpha_{j}+2 \beta_{j}\right) x_{j}+\alpha_{j}+\beta_{j}+\delta_{j} \geqslant 0 .
$$

We observe that in [3], the following monotonic polynomial $s: \mathbb{Z}^{d} \rightarrow \mathbb{Z}^{d}$ was used while constructing $\mathbb{Z}^{d}$-staircase actions:

$$
s(x)_{i}=x_{i}\left(x_{1}+\cdots+x_{d}\right)-\left(x_{i}^{2}+x_{i}\right) / 2, \quad i=1, \ldots, d .
$$

(By this formula we correct a misprint on page 849 of [3].) If, moreover $d=1$, we get $s(x)=x(x-1) / 2$, i.e., the polynomial corresponding to the classical staircase shown to be mixing in [1].

Lemma 4.3. If $s_{n}$ is monotonic and

$$
\max _{x \in H_{n}}\left\|s_{n}(x)\right\|_{\infty}<a_{n} / 2
$$

then (1.4) holds.

Proof. Suppose that the contrary holds. Then there exist $x \neq y \in H_{n}$ and $f, f^{\prime} \in F_{n}$ such that $a_{n} x+s_{n}(x)+f=$ $a_{n} y+s_{n}(y)+f^{\prime}$. Since

$$
F_{n}-F_{n}=\left\{g \in G \mid\|g\|_{\infty}<a_{n}\right\},
$$

it follows that $\left\|a_{n}(x-y)+s_{n}(x)-s_{n}(y)\right\|_{\infty}<a_{n}$. If $\|x-y\|_{\infty} \geqslant 2$ then we get a contradiction with (4.1). If $\|x-y\|_{\infty}=1$ then we get a contradiction with the fact that $s_{n}$ is monotonic.

Now suppose that $s_{n}=s$ for all $n \geqslant 0$, where $s$ is a monotonic polynomial of degree $l>1$. Let $\lambda_{G}$ stand for the direct product of the Lebesgue measure on $\mathbb{R}^{d_{1}}$ and the 'counting' measure on $\mathbb{Z}^{d_{2}}$. Let us check (1.6). Since $\lambda_{G}\left(F_{n}\right)=a_{n}^{d}$ and

$$
\lambda_{G}\left(F_{n+1} \backslash\left(F_{n}+C_{n+1}\right)\right)=\left(r_{n} a_{n}+\max _{h \in H_{n}}\|s(h)\|_{\infty} \pm 1\right)^{d}-\left(r_{n} a_{n}\right)^{d},
$$

we see that (1.6) is satisfied if and only if

$$
\sum_{n=1}^{\infty} \frac{\max _{h \in H_{n}}\|s(h)\|_{\infty}}{r_{n} a_{n}}<\infty
$$

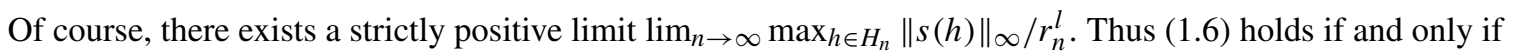

$$
\sum_{n=1}^{\infty} \frac{r_{n}^{l-1}}{a_{n}}<\infty
$$

It is easy to see that (2.9) and (2.10) are satisfied if we set

$$
Q:=\left\{h \in H \mid h \geqslant 0 \text { and }\|h\|_{\infty} \leqslant 1\right\} \text { and } Q^{\bullet}:=-Q .
$$

Proposition 4.4. Let (4.2) be satisfied. If

$$
r_{n}^{l} / a_{n} \rightarrow 0
$$

then the $(C, F)$-action $T$ associated with $\left(H_{n}, \phi_{n}, s_{n}, F_{n}\right)_{n}$ is well defined and it satisfies the restricted growth condition. Also (3.1) holds. 
Proof. Notice first that (4.4) implies (4.1) eventually. Hence (1.4) holds eventually by Lemma 4.3. Without loss of generality we may assume that it holds for all $n$. Since (1.1)-(1.3), (1.12) and (1.13) are also satisfied, it follows that the associated $(C, F)$-action $T$ of $G$ is well defined. Take a sequence $\left(h_{n}\right)_{n=1}^{\infty}$ in $H$ with $\liminf _{n \rightarrow \infty} \# H_{n}\left(h_{n}\right) / \# H_{n}>0$ (in fact, it suffices to have only $H_{n} \cap\left(H_{n}+h_{n}\right) \neq \emptyset$ eventually). It follows from (4.4) that

$$
\max _{h \in H_{n}\left(h_{n}\right)} \frac{\left\|\partial_{h_{n}} s(h)\right\|_{\infty}}{a_{n}} \leqslant \max _{h, k \in H_{n}} \frac{\|s(h)-s(k)\|_{\infty}}{a_{n}} \rightarrow 0 .
$$

On the other hand, for any $b \in G$ with $\|b\|_{\infty}<a_{n}$, we have

$$
\frac{\lambda_{G}\left(F_{n} \backslash\left(F_{n}+b\right)\right)}{\lambda_{G}\left(F_{n}\right)} \leqslant \frac{a_{n}^{d}-\left(a_{n}-\|b\|_{\infty}\right)^{d}}{a_{n}^{d}} \leqslant \frac{d\|b\|_{\infty}}{a_{n}} .
$$

Hence

$$
\frac{1}{\# H_{n}} \sum_{h \in H_{n}\left(h_{n}\right)} \frac{\lambda_{G}\left(F_{n} \backslash F_{n}\left(\partial_{h_{n}} s(h)\right)\right)}{\lambda_{G}\left(F_{n}\right)} \leqslant \max _{h \in H_{n}\left(h_{n}\right)} \frac{\lambda_{G}\left(F_{n} \backslash\left(F_{n}+\partial_{h_{n}} s(h)\right)\right)}{\lambda_{G}\left(F_{n}\right)} \rightarrow 0,
$$

i.e., (2.3) holds. (3.1) is verified in a similar way.

We see, in particular, that if $r_{n}$ is of sub-exponential growth, i.e., $r_{n} / \xi^{n} \rightarrow 0$ for some $\xi>1$, then both (4.2) and (4.4) hold and hence $T$ is well defined for any monotonic $s$.

Now consider in more detail the case where $s: H \rightarrow G$ is a polynomial of degree 2. Given $t \in H$, we have $\partial_{t} s(h)=$ $\psi_{t}(h)+a_{t}$ for some homomorphism $\psi_{t}: H \rightarrow G$ and an element $a_{t} \in G$. It is easy to verify that $\psi_{t_{1}+t_{2}}(h)=\psi_{t_{1}}(h)+$ $\psi_{t_{2}}(h)$ for all $t_{1}, t_{2} \in H$. Hence the map $H \times H \ni(t, h) \mapsto \psi_{t}(h) \in G$ is a 'bihomomorphic' form. Then there is $\gamma>0$ such that

$$
\left\|\psi_{t}(h)\right\|_{\infty} \leqslant \gamma\|t\|_{\infty}\|h\|_{\infty} \text { for all } t, h \in H .
$$

Fix a standard 'basis' in $G$ (and $H$ ):

$$
e_{1}:=(1,0, \ldots, 0), e_{2}:=(0,1,0, \ldots, 0), \ldots, e_{d}:=(0, \ldots, 0,1) .
$$

Then we can identify $\psi_{t}$ with the corresponding $(d \times d)$-matrix with real entries for any $t \in H$.

The following statement based essentially on Theorem 3.5 provides convenient sufficient conditions for the $(C, F)$ actions under consideration to be mixing. Combined with Corollary 2.6 it will turn proofs of the main results into verifications of almost purely algebraic nature.

Proposition 4.5. Let the polynomial s be monotonic and (4.2) and (4.4) hold with $l=2$. Moreover, suppose that

(i) the action $\left(T_{\psi_{k}(h)}\right)_{h \in H}$ is ergodic for any $k \in H \backslash\{0\}$ and

(ii) $\max _{1 \leqslant i \leqslant d}\left\|\psi_{k}\left(e_{i}\right)\right\|_{\infty} \geqslant \delta\|k\|_{\infty}$ for some $\delta>0$ and every $k \in H$.

Then $T$ is mixing.

Proof. By Proposition 4.4, $T$ has restricted growth and (3.1) holds. Take a sequence $\left(h_{n}\right)_{n=1}^{\infty}$ in $H$ such that $h_{n} \neq 0$ for all $n \in \mathbb{N}$ and

$$
\liminf _{n \rightarrow \infty} \# H_{n}\left(h_{n}\right) / \# H_{n}>0 .
$$

In view of Theorem 3.5, it suffices to show that $\mathcal{M}_{\psi_{h_{n}}, H_{n}\left(h_{n}\right)} \rightarrow P_{0}$ strongly. Suppose first that $h_{n}=k$ for some $k \in H$ and all $n$. Then just use (i) and apply the mean ergodic theorem to the action $\left(T_{\psi_{k}(h)}\right)_{h \in H}$ to conclude that $\mathcal{M}_{\psi_{k}, H_{n}(k)} \rightarrow P_{0}$. Hence it remains to consider only the sequences $\left(h_{n}\right)_{n=1}^{\infty}$ with $h_{n} \rightarrow \infty$ in $H$. Let $i(k)$ stand for an index at which the maximum in (ii) is attained. Then

$$
\left\|\psi_{h_{n}}\left(e_{i\left(h_{n}\right)}\right)\right\|_{\infty} \geqslant \delta\left\|h_{n}\right\|_{\infty} \text {. }
$$

Since $H_{n}\left(h_{n}\right)$ is a parallelepiped in $\mathbb{Z}_{+}^{d}$, there exist an element $h_{n}^{\prime} \in H$, a positive integer $r_{n}^{\prime} \leqslant r_{n}$ and two parallelepipeds $J_{n} \subset \mathbb{Z}_{+}^{i\left(h_{n}\right)-1}$ and $J_{n}^{\prime} \subset \mathbb{Z}_{+}^{d-i\left(h_{n}\right)}$ such that

$$
H_{n}\left(h_{n}\right)+h_{n}^{\prime}=J_{n} \times\left\{0,1, \ldots, r_{n}^{\prime}-1\right\} \times J_{n}^{\prime} .
$$


It follows from (4.6) that there exist two reals $\delta^{\prime}>0$ and $\delta^{\prime \prime} \leqslant 1$ such that

$$
r_{n}^{\prime} / r_{n} \geqslant \delta^{\prime} \text { and }\left\|h_{n}\right\|_{\infty} / r_{n} \leqslant \delta^{\prime \prime} \quad \text { eventually. }
$$

We set $V_{n}:=H_{n}\left(h_{n}\right)+h_{n}^{\prime}$ and $g_{n}:=\psi_{h_{n}}\left(e_{i\left(h_{n}\right)}\right)$. By (4.7), $\left\|g_{n}\right\|_{\infty} \rightarrow \infty$ (recall that $\left.h_{n} \rightarrow \infty\right)$. Find $p_{n} \in \mathbb{N}$ with $g_{n} \in F_{p_{n}+1}^{\bullet} \backslash F_{p_{n}}^{\bullet}$. Then, of course, $p_{n} \rightarrow \infty$. Let $m_{n}$ be the smallest positive integer such that $m_{n} g_{n} \notin F_{p_{n}+1}^{\bullet}$. Then

$$
m_{n} g_{n}=\left(m_{n}-1\right) g_{n}+g_{n} \in F_{p_{n}+1}^{\bullet}+F_{p_{n}+1}^{\bullet} .
$$

Since $\left\{g \in G \mid\|g\|_{\infty} \leqslant a_{m}\right\} \supset F_{m}^{\bullet} \supset\left\{g \in G \mid\|g\|_{\infty}<a_{m}\right\}$ for all $m \in \mathbb{N}$, we have

$$
a_{p_{n}} \leqslant\left\|g_{n}\right\|_{\infty} \leqslant a_{p_{n}+1} \quad \text { and } \quad a_{p_{n}+1} \leqslant m_{n}\left\|g_{n}\right\|_{\infty} \leqslant 2 a_{p_{n}+1} .
$$

Moreover, it is straightforward (see (4.3)) that

$$
\underbrace{Q+\cdots+Q}_{l \text { times }}=\left\{h \in H \mid h \geqslant 0 \text { and }\|h\|_{\infty} \leqslant l\right\}
$$

and

$$
Q_{+} \cap\left\{h \in H \mid\|h\|_{\infty}=1 \text { and }-h \geqslant 0\right\}=\emptyset .
$$

Recall that $Q_{+}$denotes the minimal (finite) subset of $H$ such that $\left(F_{n}^{\bullet}+F_{n}^{\bullet}\right) \backslash F_{n}^{\bullet} \subset \phi_{n}\left(Q_{+}\right)+F_{n}$ for all $n \in \mathbb{N}$. Hence

$$
-l Q_{+} \cap(\underbrace{Q+\cdots+Q}_{l \text { times }})=\emptyset .
$$

By (i) and Corollary 2.6(i), the sequence $\left(\phi_{n}(k)\right)_{n=1}^{\infty}$ is mixing for every $0 \neq k \in H$. Thus we may apply Lemma 2.9 and conclude that the sequence $\left(l_{n} g_{n}\right)_{n=1}^{\infty}$ is mixing for every $l \in \mathbb{N}$. Notice that

$$
\mathcal{M}_{\psi_{h_{n}}, H_{n}\left(h_{n}\right)}=\mathcal{M}_{\psi_{h_{n}}, V_{n}} U_{n}=U_{n} \mathcal{M}_{\psi_{h_{n}}, V_{n}},
$$

where $U_{n}$ is the unitary operator given by $U_{n} f:=f \circ T_{\psi_{h_{n}}\left(-h_{n}^{\prime}\right)}$. Therefore, $\mathcal{M}_{\psi_{h_{n}}, H_{n}\left(h_{n}\right)} \rightarrow P_{0}$ strongly if and only if $\mathcal{M}_{\psi_{h_{n}} V_{n}} \rightarrow P_{0}$ strongly as $n \rightarrow \infty$. We set $I_{n}:=\left\{0, e_{i\left(h_{n}\right)}, \ldots,\left(r_{n}^{\prime}-1\right) e_{i\left(h_{n}\right)}\right\}$. To complete the proof it remains only to establish that $m_{n} / \# I_{n} \rightarrow 0$ and apply Lemma 3.10. By (4.5),

$$
\left\|g_{n}\right\|_{\infty}=\left\|\psi_{h_{n}}\left(e_{i\left(h_{n}\right)}\right)\right\|_{\infty} \leqslant \gamma\left\|h_{n}\right\|_{\infty}
$$

eventually in $n$. Using that, (4.8) and (4.9) we obtain

$$
\frac{m_{n}}{r_{n}} \leqslant \frac{2 \gamma \delta^{\prime \prime} a_{p_{n}+1}}{\left\|g_{n}\right\|_{\infty}^{2}} \leqslant 2 \gamma \delta^{\prime \prime} \frac{a_{p_{n}+1}}{a_{p_{n}}^{2}}
$$

for all large enough $n$. It follows (use also (4.8) plus (4.2))

$$
\lim _{n \rightarrow \infty} \frac{m_{n}}{r_{n}^{\prime}} \leqslant \frac{1}{\delta^{\prime}} \lim _{n \rightarrow \infty} \frac{m_{n}}{r_{n}} \leqslant \frac{2 \gamma \delta^{\prime \prime}}{\delta^{\prime}} \lim _{n \rightarrow \infty} \frac{a_{p_{n}+1}}{a_{p_{n}}^{2}}=\frac{2 \gamma \delta^{\prime \prime}}{\delta^{\prime}} \lim _{n \rightarrow \infty} \frac{r_{p_{n}}}{a_{p_{n}}}=0 .
$$

(Notice that it follows from (1.6) that $\lim _{n \rightarrow \infty} a_{n+1} /\left(a_{n} r_{n}\right)=1$.)

Fix a family of reals $\xi_{1}, \ldots, \xi_{m}$. For a non-empty subset $J \subset\{1, \ldots, m\}$, we let $\xi_{J}:=\prod_{i \in J} \xi_{i}$. We also let $\xi_{\emptyset}:=1$.

Definition 4.6. If the family of reals $\xi_{J}, J$ runs all the subsets of $\{1, \ldots, m\}$, is independent over $\mathbb{Q}$ then we say that $\xi_{1}, \ldots, \xi_{m}$ is good.

It is clear that any subfamily of a good family is good. Moreover, given non-zero rationals $q_{1}, \ldots, q_{m}$, the family $q_{1} \xi_{1}, \ldots, q_{m} \xi_{m}$ is good if and only if so is $\xi_{1}, \ldots, \xi_{m}$.

We also let $\mathbb{R}^{J}:=\left\{g=\left(g_{1}, \ldots, g_{d}\right) \in \mathbb{R}^{d} \mid g_{i}=0\right.$ for all $\left.i \notin J\right\}$. In a similar way we define $\mathbb{Z}^{J}$. 
Lemma 4.7. Let $\xi_{1}, \ldots, \xi_{m}$ be a good family. Then

$$
\operatorname{det}\left(\begin{array}{cccc}
q_{1,1}+\xi_{1} & q_{1,2} & \ldots & q_{1, m} \\
q_{2,1} & q_{2,2}+\xi_{2} & \ldots & q_{2, m} \\
\vdots & \vdots & \ddots & \vdots \\
q_{1, m} & q_{2, m} & \ldots & q_{m, m}+\xi_{m}
\end{array}\right) \neq 0
$$

for any $q_{i, j} \in \mathbb{Q}, i, j=1, \ldots, m$.

Proof. It is enough to notice that the determinant equals to $\sum_{J \subset\{1, \ldots, m\}} r_{J} \xi_{J}$ with some coefficients $r_{J} \in \mathbb{Q}$. If the determinant vanishes then $r_{J}=0$ for all $J$ by the definition of a good family. However, it is easy to see that $\xi_{\{1, \ldots, m\}}=1$, a contradiction.

We also state without proof a couple of well known facts.

\section{Lemma 4.8.}

(i) A weakly mixing action of a l.c.s.c. Abelian group is totally ergodic.

(ii) Let $V$ and $V^{\prime}$ be two mutually commuting actions of l.c.s.c. Abelian groups $F$ and $F^{\prime}$ respectively. If $V$ is weakly mixing and $V^{\prime}$ is ergodic then $V^{\prime}$ is weakly mixing.

Now we are ready to prove the main results of the paper. We consider first the case where $d_{2}=0$ and $d_{1}>1$.

Theorem 4.9. Let $G=\mathbb{R}^{d}, d>1$, and (4.2) and (4.3) hold. Let $s$ be the polynomial from Example 4.2 and let the parameters $\alpha_{i}, \beta_{i}$ satisfy the following additional conditions: $\alpha_{i} \in \mathbb{Q}$ for all $i=1, \ldots, d$ and the family $\alpha_{1}+2 \beta_{2}, \ldots, \alpha_{d}+2 \beta_{d}$ is good. Put $s_{n}:=s$ for all $n \in \mathbb{N}$. Then the rank-one action $T$ of $G$ associated with $\left(H_{n}, \phi_{n}, s_{n}, F_{n}\right)_{n}$ is mixing.

Proof. It is easy to calculate that

$$
\psi_{e_{i}}=\left(\begin{array}{ccccccc}
\alpha_{1} & & & & & & \\
& \ddots & & & & & \\
& & \alpha_{i-1} & & & & \\
\alpha_{i} & \ldots & \alpha_{i} & 2\left(\alpha_{i}+\beta_{i}\right) & \alpha_{i} & \ldots & \alpha_{i} \\
& & & & \alpha_{i+1} & & \\
& & & & & \ddots & \\
& & & & & & \alpha_{d}
\end{array}\right),
$$

where the entries outside the main diagonal and the $i$-th line are zero, $i=1, \ldots, d$.

We first claim that the group generated by $\bigcup_{i, j=1}^{d} \psi_{e_{i}}\left(e_{j}\right)$ is dense in $G$. For a real $x \in \mathbb{R}$, denote the fractional part of $x$ by $\langle x\rangle$. Identify $\mathbb{T}$ with the interval $[0,1)$ endowed with addition mod 1 . Since $\psi_{e_{i}}\left(e_{i}\right)=2\left(\alpha_{i}+\beta_{i}\right) e_{i}$ and $\alpha_{i}+\beta_{i} \neq 0$, the map

$$
\pi: G \ni\left(x_{1}, \ldots, x_{d}\right) \mapsto\left(\left\langle\frac{x_{1}}{2\left(\alpha_{1}+\beta_{1}\right)}\right\rangle, \ldots,\left\langle\frac{x_{d}}{2\left(\alpha_{d}+\beta_{d}\right)}\right\rangle\right) \in \mathbb{T}^{d}
$$

is a quotient of $G$ by the lattice generated by $\bigcup_{i=1}^{d} \psi_{e_{i}}\left(e_{i}\right)$. We see that for $i \neq 1$,

$$
\pi\left(\psi_{e_{1}}\left(e_{i}\right)\right)=(\underbrace{\left\langle\frac{\alpha_{1}}{2\left(\alpha_{1}+\beta_{1}\right)}\right\rangle, 0, \ldots, 0,\left\langle\frac{\alpha_{i}}{2\left(\alpha_{i}+\beta_{i}\right)}\right\rangle}_{i}, 0, \ldots, 0) .
$$

Since $\alpha_{1}+\beta_{1} \notin \mathbb{Q}$ and $\left(\alpha_{i}+\beta_{i}\right) /\left(\alpha_{1}+\beta_{1}\right) \notin \mathbb{Q}$ for $i=2, \ldots, d$, the group generated by $\pi\left(\psi_{e_{1}}\left(e_{i}\right)\right)$ is dense in the subgroup

$$
\underbrace{\mathbb{T} \times\{0\} \times \cdots \times\{0\} \times \mathbb{T}}_{i} \times\{0\} \times \cdots \times\{0\} \subset \mathbb{T} .
$$


Thus our first claim follows.

Now let us verify conditions (i) and (ii) from Proposition 4.5.

It follows from (4.10) that det $\psi_{e_{i}} \neq 0$. Hence $\psi_{e_{i}}(H)$ is a lattice in $G$. Then by Corollary 2.6(iii), the sequence $\left(\phi_{n}\left(e_{i}\right)\right)_{n=1}^{\infty}$ is uniformly mixing. Since $\phi_{n}\left(e_{i}\right)=a_{n} e_{i}$, it follows that the transformation $T_{e_{i}}$ is weakly mixing for every $i=1, \ldots, m$. By Lemma 4.8(ii), the action $\left(T_{g}\right)_{g \in \mathbb{R}^{J}}$ is also weakly mixing for any non-empty subset $J \subset\{1, \ldots, m\}$. Now take any $0 \neq t=\left(t_{1}, \ldots, t_{d}\right) \in H$. If $\operatorname{det} \psi_{t} \neq 0$ then $\psi_{t}(H)$ is a lattice in $G$. By Corollary 2.6(ii), $\left(T_{\psi_{t}(h)}\right)_{h \in H}$ is ergodic, as desired. Consider now the second case when $\operatorname{det} \psi_{t}=0$. Since $\psi_{t}=t_{1} \psi_{e_{1}}+\cdots+t_{d} \psi_{e_{d}}$, it follows from (4.10) that

$$
\psi_{t}=\left(\begin{array}{cccc}
\left(\alpha_{1}+2 \beta_{1}\right) t_{1}+\alpha_{1} u & \alpha_{1} t_{1} & \ldots & \alpha_{1} t_{1} \\
\alpha_{2} t_{2} & \left(\alpha_{2}+2 \beta_{2}\right) t_{2}+\alpha_{2} u & \ldots & \alpha_{2} t_{2} \\
\vdots & \vdots & \ddots & \vdots \\
\alpha_{d} t_{d} & \alpha_{d} t_{d} & \ldots & \left(\alpha_{d}+2 \beta_{d}\right) t_{d}+\alpha_{d} u
\end{array}\right) \text {, }
$$

where $u:=\sum_{i=1}^{d} t_{i}$. Let $J:=\left\{i \mid t_{i} \neq 0\right\}$. It follows immediately from Lemma 4.7 that there exists $i \notin J$. Then for any $j \neq i$, the $j$-th element of the $i$-th line of $\psi_{t}$ is 0 . Crossing out the $i$-th line and the $i$-column for all $i \notin J$ we obtain a $(\# J \times \# J)$-matrix $M$ and det $\psi_{t}=u^{d-\# J} \prod_{i \notin J} \alpha_{i} \operatorname{det} M$. It is easy to see that Lemma 4.7 is applicable to $M$. Hence $\operatorname{det} M \neq 0$. Since $\operatorname{det} \psi_{t}=0$, it follows that $u=0$. Hence for any $j \notin J$, the entire $i$-th line in $\psi_{t}$ vanishes. Therefore $\psi_{t}(H) \subset \mathbb{R}^{J}$. Moreover, $\psi_{t}(H)$ is a lattice in $\mathbb{R}^{J}$ since det $M \neq 0$. By Lemma 4.8(i), the action $\left(T_{\psi_{t}(h)}\right)_{h \in H}$ is ergodic. Thus, Proposition 4.5(i) holds. The other condition of that theorem is much easier to verify. Let $\|t\|_{\infty}=\left|t_{j}\right|$ for some $j$. Take any $l \neq j$. Then we have

$$
\left\|\psi_{t}\left(e_{l}\right)\right\|_{\infty} \geqslant \alpha_{j}\left|t_{j}\right| \geqslant \min _{1 \leqslant i \leqslant d} \alpha_{i} \cdot\|t\|_{\infty} .
$$

It is easy to understand that the above construction does not work in case $G=\mathbb{R}$ since the first claim of the proof fails. However, only a slight 'complication' of the construction is needed to cover the exceptional case. We outline it briefly. Let $S$ be a finite family of monotonic polynomials from $H$ to $G=\mathbb{R}^{d_{1}} \times \mathbb{Z}^{d_{2}}$ of degree $l>1$. Let $\left(s_{n}\right)_{n=1}^{\infty}$ be a sequence of elements of $S$ such that every element of $S$ occurs in $\left(s_{n}\right)_{n=1}^{\infty}$ infinitely many times. Suppose that (4.2) and (4.4) are satisfied. Define $H_{n}, \phi_{n}, F_{n}$ as above. Then the $(C, F)$-action $T$ associated with $\left(H_{n}, \phi_{n}, s_{n}, F_{n}\right)_{n}$ is well defined and it satisfies the restricted growth condition (the same proof as in Proposition 4.4 works as well to establish this fact). Let us assume now that $l=2$. For any $s \in S$, let

$$
\partial_{t} s(h)=\psi_{t}^{s}(h)+a_{t}^{s} \quad \text { for all } t, h \in H,
$$

where $\psi_{t}^{s}: H \rightarrow H$ is a homomorphism and $a_{t}^{s}$ an element of $H$. Suppose, in addition, that

(i) the action $\left(T_{\psi_{k}^{s}(h)}\right)_{h \in H}$ is ergodic for any $k \in H \backslash\{0\}$ and $s \in S$ and

(ii) $\min _{s \in S} \max _{1 \leqslant i \leqslant d}\left\|\psi_{k}^{s}\left(e_{i}\right)\right\|_{\infty} \geqslant \delta\|k\|_{\infty}$.

Then repeating the proof of Proposition 4.5 almost verbatim one can show that $T$ is mixing. Now let $G=\mathbb{R}^{d}$ for any $d>0$. Suppose that every polynomial from $S$ satisfies the conditions of Theorem 4.9. Then slightly modifying the proof of this theorem one can establish that $T$ is mixing whenever

$$
\text { the group generated by } \bigcup_{s \in S} \bigcup_{i, j=1}^{d} \psi_{e_{i}}^{s}\left(e_{j}\right) \text { is dense in } G \text {. }
$$

The main point of this modification is to replace the references to Corollary 2.6 with the references to Remark 2.7. If $d>1$ then (4.11) is satisfied (see the proof of Theorem 4.9). The following theorem provides an example where (4.11) holds in case $d=1$.

Theorem 4.10. Let $G=\mathbb{R}$ and (4.2) and (4.3) hold with $l=2$. Let

$$
s(x):=\alpha x^{2}+\beta x, \quad \tilde{s}(x):=\tilde{\alpha} x^{2}+\tilde{\beta} x \text { at all } x \in \mathbb{Z},
$$

where $\alpha$ and $\tilde{\alpha}$ are rationally independent positive reals and $\alpha+\beta \geqslant 0, \tilde{\alpha}+\tilde{\beta} \geqslant 0$. Set $s_{n}:=s$ for even $n$ and $s_{n}:=\tilde{s}$ for odd $n$. Then the $(C, F)$-action of $\mathbb{R}$ associated with $\left(H_{n}, \phi_{n}, s_{n}, F_{n}\right)_{n}$ is mixing. 
Proof. In view of the reasoning preceding the statement of the theorem it suffices to notice that $s$ and $\tilde{s}$ are both non-negative and non-decreasing on $\mathbb{Z}_{+}$and (4.11) is satisfied since $\psi_{e_{1}}^{s}\left(e_{1}\right)=2 \alpha$ and $\psi_{e_{1}}^{\tilde{s}}\left(e_{1}\right)=2 \tilde{\alpha}$.

Now we pass to the case $G=\mathbb{Z}^{d}$.

Theorem 4.11. Let $G=\mathbb{Z}^{d}$ and (4.2) and (4.4) hold with $l=2$. Let $s$ be the polynomial from Example 4.2 with $\alpha_{1}=\cdots=\alpha_{d}=1$ and $\beta_{1}=\cdots=\beta_{d}=-0.5$. Put $s_{n}:=s$ for all $n \in \mathbb{N}$. Then the rank-one action $T$ of $G$ associated with $\left(H_{n}, \phi_{n}, s_{n}, F_{n}\right)_{n}$ is mixing.

Proof. As in the proof of Theorem 4.9 it suffices to show that the group generated by $\bigcup_{i, j=1}^{d} \psi_{e_{i}}\left(e_{j}\right)$ is ( $=$ dense in) $G$ and the conditions (i) and (ii) of Proposition 4.5 are satisfied. The first claim is trivial since $\psi_{e_{i}}\left(e_{i}\right)=e_{i}$ for all $i=$ $1, \ldots, d$ (see (4.10)). Now take any $t=\left(t_{1}, \ldots, t_{d}\right) \in G$ and set $J(t):=\left\{i \mid t_{i} \neq 0\right\}$. We will check Proposition 4.5(i) by induction in \#J(t).

If $\# J(t)=1$ then $t=t_{i} e_{i}$ for some $1 \leqslant i \leqslant d$ and a non-zero integer $t_{i}$. Hence $\operatorname{det} \psi_{t}=t_{i}^{d} \operatorname{det} \psi_{e_{i}} \neq 0$. It remains to apply Corollary 2.6(ii).

Suppose now that there exists $p<d$ such that the action $\left(T_{\psi_{t}(h)}\right)_{h \in H}$ is ergodic for any $t \in H$ with $1 \leqslant \# J(t) \leqslant p$. Take any $t \in H$ with $\# J(t)=p+1$. We are going to show that the action $\left(T_{\psi_{t}(h)}\right)_{h \in H}$ is ergodic. For convenience, let us assume that $J(t)=\{1, \ldots, p+1\}$. (In the general case one can argue in a similar way.) Then

$$
\psi_{t}=\left(\begin{array}{ccccccc}
\sum_{i=1}^{p+1} t_{i} & t_{1} & \ldots & t_{1} & t_{1} & \ldots & t_{1} \\
t_{2} & \sum_{i=1}^{p+1} t_{i} & \ldots & t_{2} & t_{2} & \ldots & t_{2} \\
\vdots & \vdots & \ddots & \vdots & \vdots & \ddots & \vdots \\
t_{p+1} & t_{p+1} & \ldots & \sum_{i=1}^{p+1} t_{i} & t_{p+1} & \ldots & t_{p+1} \\
0 & 0 & \ldots & 0 & \sum_{i=1}^{p+1} t_{i} & \ldots & 0 \\
\vdots & \vdots & \ddots & \vdots & \vdots & \ddots & \vdots \\
0 & 0 & \ldots & 0 & 0 & \ldots & \sum_{i=1}^{p+1} t_{i}
\end{array}\right) .
$$

We consider now three possible cases. Denote by $A$ the $(p+1) \times(p+1)$-submatrix of $\psi_{t}$ standing at the upper left corner.

Case 1. If $\sum_{i=1}^{p+1} t_{i} \neq 0$ and $\operatorname{det} A \neq 0$ then $\operatorname{det} \psi_{t}=\left(\sum_{i=1}^{p+1} t_{i}\right)^{d-p-1} \operatorname{det} A \neq 0$. Hence $\psi_{t}(H)$ is of finite index in $G$ and we are done (just apply Corollary 2.6(ii)).

Case 2. If $\sum_{i=1}^{p+1} t_{i} \neq 0$ but det $A=0$ then it is easy to verify that rk $A \geqslant 2$. Hence rk $\psi_{t} \geqslant d-(p+1)+2>d-p$. Therefore

$$
\psi_{t}(H) \cap \mathbb{Z}^{J(t)} \neq\{0\} .
$$

On the other hand, it follows from the inductive assumption and Corollary 2.6(i) that the sequence $\left(\phi_{n}(h)\right)_{n=1}^{\infty}$ is mixing for $T$ for any non-zero $h \in \mathbb{Z} e_{1}+\cdots+\mathbb{Z} e_{p}$. Hence the transformation $T_{h}$ is weakly mixing for all such $h$ (recall that $\left.\phi_{n}(h)=a_{n} h\right)$. Thus the transformation group $\left(T_{\psi_{t}(h)}\right)_{h \in H}$ contains a weakly mixing transformation and therefore it is ergodic.

Case 3. If $\sum_{i=1}^{p+1} t_{i}=0$ then it is easy to see that $\psi_{t}(H) \subset \mathbb{Z}^{J(t)}$. Moreover,

$$
\operatorname{det} A=t_{1} \cdots t_{p+1} \operatorname{det}\left(\begin{array}{ccccc}
0 & 1 & 1 & \ldots & 1 \\
1 & 0 & 1 & \ldots & 1 \\
\vdots & \vdots & \vdots & \ddots & \vdots \\
1 & 1 & 1 & \ldots & 0
\end{array}\right) \neq 0
$$

Hence $\psi_{t}(H)$ is of finite index in $\mathbb{Z}^{J(h)}$. Since the transformation $T_{e_{1}}$ is weakly mixing, the action $\left(T_{g}\right)_{g \in \mathbb{Z}^{J(h)}}$ is weakly mixing by Lemma 4.8(ii). It remains to make use of Lemma 4.8(i). 
The condition (ii) of Proposition 4.5 is checked in a straightforward way (as in the proof of Theorem 4.9).

Remark 4.12. Note that if we put $\gamma_{i}=\delta_{i}=0$ for all $i$ in the statement of Theorem 4.10 (see the definition of $s$ in Example 4.2) then we obtain exactly the main result of [3]. However, it was proved there completely only for $G=\mathbb{Z}^{2}$. Roughly speaking, it was assumed implicitly in [3] that det $\psi_{t} \neq 0$ for all $t \in \mathbb{Z}^{d} \backslash\{0\}$. While being the case for $d=2$, it is no longer true for $d>2$ (see Cases 2 and 3 in the proof of Theorem 4.10).

Combining the arguments from Theorems 4.9-4.11, one can prove the following.

Theorem 4.13. Let $G=\mathbb{R}^{d_{1}} \times \mathbb{Z}^{d_{2}}$ and (4.2) and (4.4) hold. Let $\tilde{s}_{n}: \mathbb{Z}^{d_{1}} \rightarrow \mathbb{R}^{d_{1}}$ and $\hat{s}_{n}: \mathbb{Z}^{d_{2}} \rightarrow \mathbb{Z}^{d_{2}}$ stand for the sequences of polynomials defined in the statements of Theorem 4.9 if $d_{1}>1$ (or Theorem 4.10 if $d_{1}=1$ ) and Theorem 4.11 respectively. We set

$$
s_{n}\left(t_{1}, \ldots, t_{d_{1}+d_{2}}\right):=\left(\tilde{s}_{n}\left(t_{1}, \ldots, t_{d_{1}}\right), \hat{s}_{n}\left(t_{d_{1}+1}, \ldots, t_{d_{1}+d_{2}}\right)\right)
$$

for all $\left(t_{1}, \ldots, t_{d_{1}+d_{2}}\right) \in H=\mathbb{Z}^{d_{1}} \times \mathbb{Z}^{d_{2}}$. Then the rank-one $(C, F)$-action of $G$ associated with $\left(H_{n}, \phi_{n}, s_{n}, F_{n}\right)_{n}$ is mixing.

Now we consider a more general case where the 'spacer mapping' $s: H \rightarrow G$ is a polynomial of arbitrary degree $l>2$. Then for any $t_{1}, \ldots, t_{l-1} \in H$, there exist a homomorphism $\psi_{t_{1}, \ldots, t_{l-1}}: H \rightarrow G$ and an element $a_{t_{1}, \ldots, t_{l-1}} \in G$ such that

$$
\partial_{t_{1}} \cdots \partial_{t_{l-1}} s(h)=\psi_{t_{1}, \ldots, t_{l-1}}(h)+a_{t_{1}, \ldots, t_{l-1}} \quad \text { for all } h \in H .
$$

It is easy to verify that the map

$$
\underbrace{H \times \cdots \times H}_{l \text { times }} \ni\left(t_{1}, \ldots, t_{l}\right) \mapsto \psi_{t_{1}, \ldots, t_{l-1}}\left(t_{l}\right) \in G
$$

is a 'polyhomomorphic' form and there exists $\gamma>0$ such that

$$
\left\|\psi_{t_{1}, \ldots, t_{l-1}}\right\|_{\infty} \leqslant \gamma\left\|t_{1}\right\|_{\infty} \cdots\left\|t_{l}\right\|_{\infty} \quad \text { for all } t_{1}, \ldots, t_{l} \in H .
$$

The following statement is a higher degree analogue of Proposition 4.5.

Proposition 4.14. Let $s: H \rightarrow G$ be a monotonic polynomial of degree $l>2$. Assume that (4.2) and (4.4) hold. Set $s_{n}:=s$ for all $n$ and denote by $T$ the $(C, F)$-action of $G$ associated with $\left(H_{n}, \phi_{n}, s_{n}, F_{n}\right)_{n}$. Suppose that

(i) the action $\left(T_{\psi_{t_{1}, \ldots, t_{l-1}}(h)}\right)_{h \in H}$ is ergodic for any family $t_{1}, \ldots, t_{l-1} \in H \backslash\{0\}$ and

(ii) $\max _{1 \leqslant i \leqslant d}\left\|\psi_{t_{1}, \ldots, t_{l-1}}\left(e_{i}\right)\right\|_{\infty} \geqslant \delta\left\|t_{1}\right\|_{\infty} \cdots\left\|t_{l-1}\right\|_{\infty}$ for some $\delta>0$ and all $t_{1}, \ldots, t_{l-1} \in H \backslash\{0\}$.

Then $T$ is mixing.

Proof. (Cf. with the proof of Proposition 4.5.) By Proposition 4.4, $T$ has restricted growth. Take a sequence $\left(h_{n}\right)_{n=1}^{\infty}$ in $H \backslash\{0\}$ such that (4.6) holds. In view of Theorem 3.5, it suffices to show that $\mathcal{M}_{s_{n}^{\prime}, H_{n}\left(h_{n}\right)} \rightarrow P_{0}$ strongly, where $s_{n}^{\prime}$ is the $h_{n}$-derivative of $s$. Applying Corollary $3.12 l-2$ times, we obtain that the latter holds whenever

$$
\mathcal{M}_{\psi_{t_{1}, \ldots, t_{l-2}, h_{n}}, H_{n}\left(h_{n}\right)} \rightarrow P_{0} \quad \text { strongly for any family } t_{1}, \ldots, t_{l-2} \in H \backslash\{0\} .
$$

Suppose first that $h_{n}=k$ for some $k \in H$ and all $n$. Then just use (i) and apply the mean ergodic theorem to the action $\left(T_{\psi_{t_{1}, \ldots, t_{l-2}, k}(h)}\right)_{h \in H}$ to deduce (4.11).

It remains to consider the second case where $h_{n} \rightarrow \infty$ in $H$. Notice first that since $\mathcal{M}_{\partial_{k} s, H_{n}(k)} \rightarrow P_{0}$ strongly, it follows from Corollary 2.3(ii) that the sequence $\left(\phi_{n}(k)\right)_{n=1}^{\infty}$ is uniformly mixing for every $k \in H \backslash\{0\}$. The rest of the argument is almost a literal repetition of that from the proof of Proposition 4.5.

As an application we construct a family of mixing $(C, F)$-actions with polynomial 'spacer map' of higher degree in the simplest case when $G=\mathbb{Z}$. 
Example 4.15. Let $G=\mathbb{Z}$. Suppose that the sequence $\left(r_{n}\right)_{n=1}^{\infty}$ is of sub-exponential growth and $\lim _{n \rightarrow \infty} r_{n}=\infty$. Then, as was noticed above, (4.2) and (4.4) hold. Let $p: \mathbb{Z} \rightarrow \mathbb{Z}$ be a polynomial of degree $l>0$ which non-decreases on $\mathbb{Z}_{+}$. Assume also that $p(0)=0$. Suppose that the image of $p$ is not contained in any proper subgroup of $\mathbb{Z}$. For instance, if $p(\mathbb{Z}) \ni 1$ then $p$ enjoys this property. Define a polynomial $s: \mathbb{Z} \rightarrow \mathbb{Z}$ by setting

$$
s(t):=p(0)+p(1)+\cdots+p(t-1) \text { for } t>0 .
$$

Clearly, $s$ is of degree $l+1$ and $s$ non-decreases on $\mathbb{Z}_{+}$. Let $s_{n}:=s$ for all $n \in \mathbb{N}$. Denote by $T$ the corresponding $\mathbb{Z}_{-}^{-}$ action. We will show that it is mixing. To this end it suffices to verify that the conditions (i) and (ii) of Proposition 4.14 hold for $T$. It is obvious that (ii) holds. The condition (i) will follow from the fact that $T$ is totally ergodic. To establish the latter we are going to apply Proposition 2.5. Let $h_{n}=1$ for all $n \in \mathbb{N}$. Then $\partial_{h_{n}} s(t)=s(t+1)-s(t)=p(t)$ at all $t \geqslant 0$. If $T$ is not totally ergodic then by Proposition 2.5 there exist $k>0$ and $j \in\{0, \ldots, k-1\}$ such that

$$
\frac{\#\left\{i \in\left\{1, \ldots, r_{n}-1\right\} \mid p(i) \equiv j \bmod k\right\}}{r_{n}-1} \rightarrow 1 \quad \text { as } n \rightarrow \infty .
$$

Hence if $n$ is large enough we can find $0<i<r_{n}-2-l$ such that

$$
p(i) \equiv p(i+1) \equiv \cdots \equiv p(i+l) \bmod k .
$$

Since $p(0)=0$ and $l$ is the degree of $p$, it follows from [16, Corollary 1.17] that $p(\mathbb{Z}) \subset k \mathbb{Z}$, a contradiction.

In particular, putting $r_{n}=n$ and $p(t)=t^{l}, t \in \mathbb{Z}$, we obtain the family of polynomial staircases first proved to be mixing in [1] (when $l=1)$ and [5] (when $l>1$ ).

\section{Entropic properties of rank-one actions}

Let $G=\mathbb{R}^{d_{1}} \times \mathbb{Z}^{d_{2}}$ with arbitrary $d_{1}, d_{2} \geqslant 0$ and $T$ a $(C, F)$-action of $G$ on a probability space $(X, \mathfrak{B}, \mu)$. We will assume that

$$
F_{n}=\left\{g \in G \mid g \geqslant 0 \text { and }\|g\|_{\infty}<a_{n}\right\}
$$

for a sequence $a_{n} \in \mathbb{R}_{+}$. This implies that $T$ is rank-one by cubes. It is well known that the entropy of any rank-one $\mathbb{Z}$-action is zero. This fact extends easily to the rank-one (by cubes) actions of any group $G$. However in case of higher dimensional groups, say $G=\mathbb{Z}^{2}$, there exist rank-one (by rectangles) actions $S=\left(S_{g}\right)_{g \in G}$ of $G$ such that the transformation $S_{(1,0)}$ is Bernoulli (see [20]). Then one has $h(S)=0$ but $h\left(S_{(1,0)}\right)>0$. We show now that this is impossible for rank-one (by cubes) actions.

Theorem 5.1. Let $T$ be a rank-one (by cubes) action of $G$. Then $h\left(T_{g}\right)=0$ for each $g \in G$.

Proof. We consider only the case $G=\mathbb{R}^{d}$ (in the general case one can argue in a similar way). Let $\left(M_{n}\right)_{n=1}^{\infty}$ be a sequence of positive reals such that

$$
\lim _{n \rightarrow \infty} \frac{M_{n}}{a_{n}}=0 \quad \text { and } \quad \lim _{n \rightarrow \infty} \frac{\log a_{n}}{M_{n}}=0 .
$$

Fix $g \in G \backslash\{0\}$ and take a family $v_{1}, \ldots, v_{d}$ of mutually orthogonal (with respect to Euclidean inner product in $\mathbb{R}^{d}$ ) vectors such that $v_{1}=g$. Consider the finest partition $\mathcal{F}$ of $G$ into parallelepipeds whose vertices belong to the lattice generated by $v_{1}, \ldots, v_{d}$. Let $\mathcal{F}_{n}$ be a maximal subset of $\mathcal{F}$ such that the atoms $i g+A, A$ runs $\mathcal{F}_{n}$ and $0 \leqslant i<M_{n}$, are mutually disjoint and all of the are contained in $F_{n}$. It follows from the first limit in (5.1) that

$$
\frac{\# \mathcal{F}_{n} \cdot M_{n} \cdot \lambda_{G}(A)}{\lambda_{G}\left(F_{n}\right)} \rightarrow 1 .
$$

Let $\mathcal{P}_{n}$ be a finite partition of $X$ consisting of the $n$-cylinders $[A]_{n}$, where $A$ runs $\mathcal{F}_{n}$ and the complement $B_{n}$ to the union of these $n$-cylinders. Then

$$
H\left(\mathcal{P}_{n}\right)=-\# \mathcal{F}_{n} \mu\left([A]_{n}\right) \log \mu\left([A]_{n}\right)-\mu\left(B_{n}\right) \log \mu\left(B_{n}\right),
$$


where $A$ is an atom of $\mathcal{F}_{n}$. Since $\mu\left(X \backslash X_{n}\right) \rightarrow 0$ and (5.2) holds, it is easy to show that the second term in the right-hand side of (5.3) goes to 0. Substituting (1.11) to (5.3) and making use of (5.2) we now obtain

$$
H\left(\mathcal{P}_{n}\right) \leqslant \# \mathcal{F}_{n} \frac{\lambda_{G}(A)}{\lambda_{G}\left(F_{n}\right)} \log \frac{\lambda_{G}\left(F_{n}\right)}{\lambda_{G}(A)}+\bar{o}(1) \leqslant \frac{2 d}{M_{n}} \log \frac{a_{n}}{\lambda_{G}(A)}+\bar{o}(1)
$$

(notice that $\lambda_{G}\left(F_{n}\right)=a_{n}^{d}$ ). It follows from the second limit in (5.1) that $H\left(\mathcal{P}_{n}\right) \rightarrow 0$ as $n \rightarrow \infty$. Denote by $\mathcal{Q}_{n}$ the finite partition $\bigvee_{i=0}^{M_{n}-1} T_{g}^{i} \mathcal{P}_{n}$ of $X$. It is easy to deduce from the definition of $\mathcal{P}_{n}$ that the sequence $\left(\mathcal{Q}_{n}\right)_{n=1}^{\infty}$ generates $\mathfrak{B}$, i.e., for any $B \in \mathfrak{B}$ and $n \in \mathbb{N}$, there exists a $\mathcal{Q}_{n}$-measurable subset $Q_{n}$ such that $\mu\left(B \Delta Q_{n}\right) \rightarrow 0$ as $n \rightarrow \infty$. Hence

$$
h\left(T_{g}\right)=\lim _{n \rightarrow \infty} h\left(T_{g}, \mathcal{Q}_{n}\right)=\lim _{n \rightarrow \infty} h\left(T_{g}, \mathcal{P}_{n}\right) \leqslant \lim _{n \rightarrow \infty} H\left(\mathcal{P}_{n}\right)=0 .
$$

We see, in particular, that for all mixing actions $T$ constructed in Theorems $4.9-4.11$ and $4.13, h\left(T_{g}\right)=0$ for all $g \in G$.

\section{References}

[1] T.M. Adams, Smorodinsky's conjecture on rank one systems, Proc. Amer. Math. Soc. 126 (1998) 739-744.

[2] T.M. Adams, N.A. Friedman, Staircase mixing, preprint, 1992.

[3] T. Adams, C.E. Silva, $\mathbb{Z}^{d}$-staircase actions, Ergodic Theory Dynam. Systems 19 (1999) 837-850.

[4] V. Bergelson, Ergodic Ramsey theory—an update, in: Ergodic Theory of $\mathbb{Z}^{d}$ Actions, Warwick, 1993-1994, in: London Math. Soc. Lecture Note Ser., vol. 228, Cambridge Univ. Press, Cambridge, 1996, pp. 1-61.

[5] D. Creutz, C.E. Silva, Mixing on a class of rank-one transformations, Ergodic Theory Dynam. Systems 24 (2004) 407-440.

[6] A.I. Danilenko, Funny rank one weak mixing for nonsingular Abelian actions, Israel J. Math. 121 (2001) 29-54.

[7] A.I. Danilenko, Infinite rank one actions and nonsingular Chacon transformations, Illinois J. Math. 48 (2004) $769-786$.

[8] A.I. Danilenko, Mixing rank-one actions for infinite sums of finite groups, Israel J. Math. 156 (2006) 341-358.

[9] A.I. Danilenko, C.E. Silva, Multiple and polynomial recurrence for Abelian actions in infinite measure, J. London Math. Soc. (2) 69 (2004) 183-200.

[10] A. del Junco, A simple map with no prime factors, Israel J. Math. 104 (1998) 301-320.

[11] A. del Junco, R. Yassawi, Multiple mixing rank one group actions, Canad. J. Math. 52 (2000) 332-347.

[12] B. Fayad, Rank one and mixing differentiable flows, Invent. Math. 160 (2005) 305-340.

[13] C. Hoffman, A loosely Bernoulli counterexample machine, Israel J. Math. 112 (1999) 237-247.

[14] S.A. Kalikow, Twofold mixing implies threefold mixing for rank one transformations, Ergodic Theory Dynam. Systems 4 (1984) $237-259$.

[15] J.L. King, Joining-rank and the structure of finite rank mixing transformations, J. Analyse Math. 51 (1988) 182-227.

[16] A. Leibman, Polynomial mappings of groups, Israel J. Math. 129 (2002) 29-60.

[17] B. Madore, Rank-one group actions with simple mixing $\mathbb{Z}$-subactions, New York J. Math. 10 (2004) 175-194.

[18] D.S. Ornstein, On the root problem in ergodic theory, in: Proc. Sixth Berkeley Symp. Math. Stat. Prob. vol. II, Univ. California, Berkeley, CA, 1970/1971, Univ. of California Press, Berkeley, CA, 1972, pp. 347-356.

[19] A. Prikhodko, Stochastic constructions of flows of rank one, Mat. Sb. 192 (2001) 61-92.

[20] D.J. Rudolph, The second centralizer of a Bernoulli shift is just its powers, Israel J. Math. 29 (1978) 167-178.

[21] D.J. Rudolph, An example of a measure preserving map with minimal self-joinings, J. Analyse Math. 35 (1979) 97-122.

[22] V.V. Ryzhikov, Mixing, rank and minimal self-joining of actions with invariant measure, Mat. Sb. 183 (1992) 133-160.

[23] V.V. Ryzhikov, Joinings and multiple mixing of the actions of finite rank, Funktsional. Anal. i Prilozhen. 27 (1993) 63-78.

[24] V.V. Ryzhikov, The Rokhlin problem on multiple mixing in the class of actions of positive local rank, Funktsional. Anal. i Prilozhen. 34 (2000) 90-93. 